\title{
Adding pseudo-observables to the four-lepton experimentalist's toolbox
}

\author{
James S. Gainer, ${ }^{a}$ Martín González-Alonso, ${ }^{b}$ Admir Greljo, ${ }^{c, d}$ Senad Isaković, ${ }^{d}$ \\ Gino Isidori, ${ }^{e}$ Andrey Korytov, ${ }^{f}$ Joseph Lykken, ${ }^{g}$ David Marzocca, ${ }^{h}$ \\ Konstantin T. Matchev, ${ }^{f}$ Predrag Milenović, ${ }^{i}$ Guenakh Mitselmakher, ${ }^{f}$ \\ Stephen Mrenna, ${ }^{j}$ Myeonghun Park, ${ }^{k}$ Aurelijus Rinkevicius ${ }^{l}$ and Nudžeim Selimović ${ }^{d}$ \\ ${ }^{a}$ Dept. of Physics and Astronomy, University of Hawaii, Honolulu, HI 96822, U.S.A. \\ ${ }^{b}$ Theoretical Physics Department, CERN, 1211 Geneva 23, Switzerland \\ ${ }^{c}$ PRISMA Cluster of Excellence and Mainz Institute for Theoretical Physics, \\ Johannes Gutenberg Universität Mainz, 55099 Mainz, Germany \\ ${ }^{d}$ Faculty of Science, University of Sarajevo, \\ Zmaja od Bosne 33-35, 71000 Sarajevo, Bosnia and Herzegovina \\ e Physik-Institut, Universität Zürich, CH-8057 Zürich, Switzerland \\ ${ }^{f}$ Physics Department, University of Florida, Gainesville, FL 32611, U.S.A. \\ ${ }^{g}$ Theoretical Physics Department, Fermilab, Batavia, IL 60510, U.S.A. \\ ${ }^{h}$ INFN, Sezione di Trieste, SISSA, Via Bonomea 265, 34136 Trieste, Italy \\ ${ }^{i}$ Experimental Physics Department, CERN, 1211 Geneva 23, Switzerland \\ ${ }^{j}$ SSE Group, Computing Division, Fermilab, Batavia, IL 60510, U.S.A. \\ ${ }^{k}$ Institute of Convergence Fundamental Studies and School of Liberal Arts, Seoultech, \\ Seoul 01811, Korea \\ ${ }^{l}$ Department of Physics, Cornell University, Ithaca, NY 14850, U.S.A. \\ E-mail: jgainer137@gmail.com, martin.gonzalez.alonso@cern.ch, \\ admir.greljo@cern.ch, senad_isakovic@hotmail.com, \\ isidori@physik.uzh.ch, korytov@phys.ufl.edu, lykken@fnal.gov, \\ marzocca@physik.uzh.ch, matchev@ufl.edu, Predrag.Milenovic@cern.ch, \\ mitselmakher@phys.ufl.edu, mrenna@fnal.gov, \\ parc.seoultech@seoultech.ac.kr, odysei@gmail.com, nudzeims@gmail.com
}

ABSTRACT: The "golden" channel, in which the newly-discovered Higgs boson decays to four leptons by means of intermediate vector bosons, is important for determining the properties of the Higgs boson and for searching for subtle new physics effects. Different approaches exist for parametrizing the relevant Higgs couplings in this channel; here we relate the use of pseudo-observables to methods based on specifying the most general amplitude or Lagrangian terms for the $H V V$ interactions. We also provide projections for sensitivity in this channel in several novel scenarios, illustrating the use of pseudo-observables, and analyze the role of kinematic distributions and (ratios of) rates in such $H \rightarrow 4 \ell$ studies.

Keywords: Higgs Physics, Beyond Standard Model

ARXIV EPRINT: 1808.00965 


\section{Contents}

1 Introduction 1

2 Frameworks, conventions, and translation 3

2.1 The "amplitude" approach 3

2.2 The "Lagrangian" approach 4

2.3 The "pseudo-observables" approach 5

2.4 Global approaches to Higgs couplings 8

$\begin{array}{llr}3 & \text { LHC projections } & 8\end{array}$

3.1 Benchmark scenarios $\quad 8$

$\begin{array}{llr}3.2 & \text { Projections using rates and kinematics } & 9\end{array}$

$\begin{array}{lll}3.3 & \text { Projections using relative normalization and kinematics } & 11\end{array}$

$\begin{array}{lll}3.4 & \text { Discussion of results } & 13\end{array}$

4 Conclusions $\quad 16$

\section{Introduction}

The discovery of a Standard Model (SM)-like Higgs boson (Higgs) at the CERN Large Hadron Collider (LHC) [1, 2] represents both the triumphant coda to one era, in which the SM was developed, tested, and confirmed, and the dawn of an exciting new era, in which physics beyond the SM (BSM) is probed, and eventually discovered. The "golden" channel, in which the Higgs decays to vector bosons (generally, $Z$ bosons) that consequently decay to leptons, has played an important role in both the discovery of the Higgs and in the subsequent measurement of its couplings. It has therefore played an important role in both experimental [1-41] and theoretical [42-135] studies of the Higgs boson.

Measuring the couplings of the Higgs boson, and thus searching for the small deviations from SM predictions that may be the hallmark of BSM physics, requires a parameterization of these couplings. We can view this parameterization as being determined by:

- Particles: first we must specify the spectrum of particles that appear, either in the final state or as intermediate resonances.

- Symmetries: next, we must determine the symmetries that the underlying theory obeys, either exactly or approximately.

- Interactions: Once the particles and symmetries have been specified, we can enumerate the possible interactions, all of which we expect to be present at some level. However, in general, there are additional considerations, such as the dimensionality of operators in an effective field theory (EFT) Lagrangian, which may allow us to consider only a finite number of terms. 
In this paper we will consider parameterizations relevant to experimental studies of the four-lepton final state at the LHC. These parametrizations are based on the choices of

1. Particles: we consider only the SM degrees of freedom, with an additional (optional) heavy $Z^{\prime}$ boson, which is introduced as a BSM example of a concrete BSM scenario that allows for different couplings to electrons and muons. In particular, we do not consider the effects of light $Z^{\prime}$ s $[28,96,98,101,103,106,108,119,120]$, dark matter, or other invisible or unidentified particles in the final state [136-144].

2. Symmetries: there have been many studies of Higgs couplings that make the (highly theoretically motivated) assumption that the Higgs is part of an $\mathrm{SU}(2)$ doublet and the only allowed operators are part of the $\mathrm{SM} \mathrm{SU}(3) \times \mathrm{SU}(2) \times \mathrm{U}(1)$ gauge symmetry. We, however, make fewer theoretical assumptions as our aim is to facilitate relatively unbiased experimental measurements. If, as expected, potential interactions, which violate the above assumptions, are absent, we have obtained additional experimental support for our pre-existing viewpoint. Thus, we will assume only Lorentz invariance and QED and QCD gauge invariance in specifying possible interactions.

3. Interactions: having made these choices of particles and symmetries, the allowed interactions are restricted. We will generally truncate the (infinite) list of possible interactions by considering operators only up to some finite mass dimension or (equivalently) amplitude structures only up to a specified power of momentum.

Experimental attempts to measure the tensor structure of Higgs to diboson couplings at the LHC (e.g., ref. [22]) have largely used two formalisms. One is the specification of the most general $H \rightarrow V V$ amplitude allowed by the relevant symmetries [58, 73]. The second is to specify the interaction Lagrangian terms consistent with these same symmetries [84, 99]. Two important points are in order: (i) these approaches have different strengths and (ii) the approaches are nearly, though not completely, equivalent in the physics they parameterize, given certain reasonable physical assumptions.

Strengths of the amplitude approach include a greater ability to parametrize absorptive (i.e., complex) contributions to the amplitude arising from loop-induced contributions from light particles running in the loop. ${ }^{1}$ The Lagrangian approach, on the other hand, is more useful when utilizing standard computational tools, such as FeynRules [145, 146], CalcHEP [147, 148], CompHEP [149-151], and MadGraph [152-155]. It may also make the connection with more comprehensive physical frameworks more straightforward.

Recently, attention has been drawn to a third approach based on the use of pseudoobservables (PO) $[156,157] .^{2}$ This approach, which was employed at the CERN Large Electron Positron (LEP) Collider [158, 159], represents a generalization of the amplitude approach and thus it shares its strengths. However, by demanding that the amplitude considered describe a transition between on-shell states, the PO method resolves several potential

\footnotetext{
${ }^{1}$ The magnitude of such contributions can be constrained using even conservative assumptions about the total Higgs width, as discussed, for example in ref. [92].

${ }^{2}$ See also A. David, A. Greljo, G. Isidori, J. Lindert, D. Marzocca and G. Passarino, Pseudo-observables, in $[128]$.
} 
ambiguities and, in particular, guarantees the gauge invariance of the quantities that are being measured. This property will become increasingly important as measurements increase in sensitivity, probing contributions from NLO effects in the SM [160-163], as well as potential new physics. The PO approach covers also BSM scenarios that affect the $H \rightarrow 4 \ell$ channel without two intermediate $Z$ or $\gamma$ states. A simple example is a heavy $Z^{\prime}$ boson in $H \rightarrow$ $Z Z^{\prime} \rightarrow 4 \ell$, but more complicated cases with additional heavy exotic intermediate states (possibly coupled in a non-universal way to SM fermions) are also covered by this approach.

Thus PO provide a useful third approach to parameterizing Higgs couplings. However, as there are also advantages to the amplitude and the Lagrangian frameworks (besides the important fact that their use by the experiments is well-established [39, 40]), it is useful to describe how the PO approach compares to these other methods. We do that in this paper. In section 2 we discuss the theoretical relationship between various parameterizations. In particular, we specify the translation between the conventions used in each of these approaches in detail, so that results obtained in the context of one framework can be easily understood in the context of the other frameworks. In section 3, we analyze the future sensitivity to Higgs pseudo-observables within specific benchmark scenarios with only two free parameters. We discuss in some detail the role of the different experimental inputs, namely the total number of events observed, the ratios between different channels and the kinematic distribution of the events. It is hoped that these results will be informative in their own right, but will also provide a useful template for future experimental studies.

\section{Frameworks, conventions, and translation}

\subsection{The "amplitude" approach}

In the "amplitude" approach $[49,58,59,73]$ that generally has been used in four-lepton studies, the most general form of the $1 \rightarrow 2$ vertex describing the decay of the putative Higgs boson to vector bosons is specified. (Of course, for the Standard Model Higgs the decay to $Z$ bosons is the most important, though interesting phenomenology can result from decays to $Z \gamma^{*}$ and $\gamma^{*} \gamma^{*}$ [100].) The resulting expression can be used to obtain helicity amplitudes for the $H \rightarrow V V$ decay; ${ }^{3}$ these can be combined with helicity amplitudes ${ }^{4}$ for the decay of the vector bosons to leptons to obtain the amplitude, and ultimately the differential cross section, for $g g \rightarrow H \rightarrow V V \rightarrow 4 \ell$ (or processes where the Higgs is produced via other mechanisms). Following ref. [22] (and references therein) we can write the general form of this amplitude as

$$
\begin{aligned}
A\left(H \rightarrow V_{1} V_{2}\right)=\frac{i}{v}\{[ & {\left[a_{1}^{V_{1} V_{2}}+\frac{\kappa_{1}^{V_{1} V_{2}} q_{1}^{2}+\kappa_{2}^{V_{1} V_{2}} q_{2}^{2}}{\left(\Lambda_{1}^{V_{1} V_{2}}\right)^{2}}\right] m_{V_{1}}^{2} \epsilon_{1}^{*} \cdot \epsilon_{2}^{*} } \\
& \left.+a_{2}^{V_{1} V_{2}} f_{\mu \nu}^{*(1)} f^{*(2), \mu \nu}+a_{3}^{V_{1} V_{2}} f_{\mu \nu}^{*(1)} \tilde{f}^{*(2), \mu \nu}\right\},
\end{aligned}
$$

\footnotetext{
${ }^{3}$ Here and below we suppress explicit indications that the vector bosons are, in general, off-shell.

${ }^{4}$ We assume that $Z$ and $\gamma$ couple to leptons as in the Standard Model. We include also a possible contribution from a $Z^{\prime}$ boson. We allow its coupling to leptons to be an arbitrary sum of vector and axial vector contributions.
} 
where $\epsilon_{i}$ is the polarization vector and $q_{i}$ is the momentum of the gauge boson labelled " $V_{i}$ " $(i=1,2)$. The contribution to the amplitude corresponding to the field strength tensor is $f^{(i) \mu \nu}=\epsilon_{i}^{\mu} q_{i}^{\nu}-\epsilon_{i}^{\nu} q_{i}^{\mu}$, and its dual is given by $\tilde{f}^{(i) \mu \nu}=\frac{1}{2} \epsilon_{\mu \nu \alpha \beta} f^{(i), \alpha \beta}$. Symmetry considerations and gauge invariance require $\kappa_{1}^{Z Z}=\kappa_{2}^{Z Z}$ and $a_{1}^{Z \gamma}, a_{1}^{\gamma \gamma}, \kappa_{1}^{\gamma \gamma}, \kappa_{2}^{\gamma \gamma}, \kappa_{1}^{Z \gamma}=0$.

We can proceed to find the helicity amplitudes that correspond to eq. (2.1) using explicit expressions for the polarization vectors $\epsilon_{1}$ and $\epsilon_{2}$. In the case of the four-chargedlepton final state, the relevant $H \rightarrow V_{1} V_{2}$ sub-amplitudes are those where $V_{1} V_{2}$ refers to $Z Z$, $Z \gamma, \gamma \gamma, Z Z^{\prime}$, etc. It is important to note that terms in the general amplitude that vanish for on-shell vector bosons do not necessarily vanish for off-shell vector bosons. In particular, one can have a $q_{\gamma}^{2} \epsilon_{\gamma}^{*} \epsilon_{Z}^{*}$ contribution, which, of course, vanishes when the photon is on-shell.

In general, the "constants" in eq. (2.1) can be taken to be Lorentz invariant functions of $q_{1}$ and $q_{2}$. (When the intermediate gauge bosons are identical, the function must also be invariant under the exchange of the labels $V_{1}$ and $V_{2}$.) An arbitrary analytic Lorentz invariant function of momenta may be expressed as

$$
f\left(q_{1}, q_{2}\right)=f_{0}+\frac{1}{\Lambda^{2}}\left(f_{21} q_{1}^{2}+f_{22} q_{2}^{2}+f_{23}\left(q_{1}+q_{2}\right)^{2}\right)+\mathcal{O}\left(\Lambda^{-4}\right),
$$

where we assume that $f\left(q_{1}, q_{2}\right)$ can be expanded in a Taylor series. When $V_{1}$ and $V_{2}$ are produced in the decay of an on-shell boson, the situation considered in this work, we can set $\left(q_{1}+q_{2}\right)^{2}=m_{H}^{2}$, where $m_{H}$ is the mass of the (Higgs) boson that decays to $V_{1}$ and $V_{2}$. Thus we see that eq. (2.1) is written to include the momentum dependence of the $a_{1}^{V V}$ through $\mathcal{O}\left(\Lambda^{-2}\right)$ terms, while only the leading constant is included for $a_{2}^{V V}$ and $a_{3}^{V V}$. This apparent inconsistency is resolved when we realize that the amplitude structures associated with $a_{2}^{V V}$ and $a_{3}^{V V}$ involve two additional powers of momenta relative to the $a_{1}^{V V}$ structure $\left(\epsilon_{1}^{*} \cdot \epsilon_{2}^{*}\right)$. Therefore, if we take the $a_{i}^{V V}$, the $\kappa_{i}$, etc. to be true constants, the expression in eq. (2.1) includes the full momentum dependence of the amplitude (in the case where the Higgs is on-shell) containing up to two powers of gauge boson momenta. We will see similar truncations in the cases of the "Lagrangian" and the "PO" approaches discussed below.

\subsection{The "Lagrangian" approach}

The $H \rightarrow V V$ interactions can also be described in the Lagrangian formalism. For $H \rightarrow 4 \ell$, the relevant interaction Lagrangian is

$$
\begin{aligned}
\mathcal{L} \supset & -\kappa_{1, Z Z} \frac{M_{Z}^{2}}{v} H Z_{\mu} Z^{\mu}-\frac{\kappa_{2, Z Z}}{2 v} H Z_{\mu \nu} Z^{\mu \nu}-\frac{\kappa_{3, Z Z}}{2 v} H Z_{\mu \nu} \tilde{Z}^{\mu \nu} \\
& +\frac{\kappa_{4, Z Z} M_{Z}^{2}}{M_{H}^{2} v} \square H Z_{\mu} Z^{\mu}+\frac{2 \kappa_{5, Z Z}}{v} H Z_{\mu} \square Z^{\mu} \\
& -\frac{\kappa_{2, Z \gamma}}{2 v} H Z_{\mu \nu} F^{\mu \nu}-\frac{\kappa_{3, Z \gamma}}{2 v} H Z_{\mu \nu} \tilde{F}^{\mu \nu}+\frac{\kappa_{5, Z \gamma} M_{Z}^{2}}{M_{H}^{2} v} H Z_{\mu} \partial_{\nu} F^{\mu \nu} \\
& -\frac{\kappa_{2, \gamma \gamma}}{2 v} H F_{\mu \nu} F^{\mu \nu}-\frac{\kappa_{3, \gamma \gamma}}{2 v} H F_{\mu \nu} \tilde{F}^{\mu \nu} \\
& -\kappa_{1, Z Z^{\prime}} \frac{M_{Z}^{2}}{v} H Z_{\mu} Z^{\prime \mu},
\end{aligned}
$$

where $F_{\mu \nu}=\partial_{\mu} A_{\nu}-\partial_{\nu} A_{\mu}$ is the QED field strength tensor; $Z_{\mu \nu}=\partial_{\mu} Z_{\nu}-\partial_{\nu} Z_{\mu}$ is defined by analogy. The dual field strength tensors $\tilde{F}$ and $\tilde{Z}$ are defined by (e.g.) $\tilde{F}_{\mu \nu}=\frac{1}{2} \epsilon_{\mu \nu \alpha \beta} F^{\alpha \beta}$, 
where $\epsilon$ is the totally antisymmetric Levi-Civita tensor. With the exception of interactions involving a $Z^{\prime}$, this Lagrangian contains all operators up to dimension five (six, if one assumes that the operators involve the vacuum expectation value of the Higgs field). We note that there are five $H Z Z$ operators in eq. (2.3), but fewer operators for $H Z \gamma, H \gamma \gamma$, and $H Z Z^{\prime}$. In the case of $H Z \gamma$ and $H \gamma \gamma$, this is due to the requirements provided by QED gauge invariance. For the $Z^{\prime}$ we have included only the lowest dimensional operator, as amplitudes involving the heavy $Z^{\prime}$ are suppressed by the large mass in the propagator. Had we chosen to consider a light $Z^{\prime}$, we would need to include the additional $H Z Z^{\prime}$ operators

$$
\begin{aligned}
\mathcal{L} \supset & -\frac{\kappa_{2, Z Z^{\prime}}}{2 v} H Z_{\mu \nu} Z^{\prime \mu \nu}-\frac{\kappa_{3, Z Z^{\prime}}}{2 v} H Z_{\mu \nu} \tilde{Z}^{\prime \mu \nu} \\
& +\frac{\kappa_{4, Z Z^{\prime}} M_{Z}^{2}}{M_{H}^{2} v} \square H Z_{\mu} Z^{\prime \mu}+\frac{2 \kappa_{5, Z Z^{\prime}}}{v} H Z_{\mu} \square Z^{\prime \mu},
\end{aligned}
$$

the $H Z^{\prime} \gamma$ operators

$$
\mathcal{L} \supset-\frac{\kappa_{2, Z^{\prime} \gamma}}{2 v} H Z_{\mu \nu}^{\prime} F^{\mu \nu}-\frac{\kappa_{3, Z^{\prime} \gamma}}{2 v} H Z_{\mu \nu}^{\prime} \tilde{F}^{\mu \nu}+\frac{\kappa_{5, Z^{\prime} \gamma} M_{Z}^{2}}{M_{H}^{2} v} H Z_{\mu}^{\prime} \partial_{\nu} F^{\mu \nu}
$$

and the $H Z^{\prime} Z^{\prime}$ operators

$$
\begin{aligned}
\mathcal{L} \supset & -\kappa_{1, Z^{\prime} Z^{\prime}} \frac{M_{Z}^{2}}{v} H Z_{\mu}^{\prime} Z^{\prime \mu}-\frac{\kappa_{2, Z^{\prime} Z^{\prime}}}{2 v} H Z_{\mu \nu}^{\prime} Z^{\prime \mu \nu}-\frac{\kappa_{3, Z^{\prime} Z^{\prime}}}{2 v} H Z_{\mu \nu}^{\prime} \tilde{Z}^{\prime \mu \nu} \\
& +\frac{\kappa_{4, Z^{\prime} Z^{\prime}} M_{Z}^{2}}{M_{H}^{2} v} \square H Z_{\mu}^{\prime} Z^{\prime \mu}+\frac{2 \kappa_{5, Z^{\prime} Z^{\prime}}}{v} H Z_{\mu}^{\prime} \square Z^{\prime \mu} .
\end{aligned}
$$

However, assuming the $Z^{\prime}$ mass to be comparable to the cutoff of the theory, we can take all of the operators in eqs. (2.4)-(2.6) to be higher dimensional and therefore ignore them. ${ }^{5}$ For on-shell Higgs studies (which are our focus here), the $(\square H) Z_{\mu} Z^{\mu}$ operator can be absorbed into the $H Z_{\mu} Z^{\mu}$ operator, though this will not be the case when the Higgs is off-shell [99]. With the caveat that the coefficients $\left\{\kappa_{i, V V}\right\}$ in eq. (2.3) are real, there is a one-to-one mapping between the coefficients in eqs. (2.3) and (2.1), as shown explicitly in table 1 (see also ref. [99]).

While we assume that $Z$ bosons and photons couple to leptons as in the SM, we can make no such assumptions about the interaction of the $Z^{\prime}$ with leptons. Thus, to fully characterize $H \rightarrow 4 \ell$ decays, we parameterize the $Z^{\prime}$ interaction to SM leptons with

$$
\mathcal{L} \supset \sum_{\ell=\ell_{L}, \ell_{R}} g_{Z^{\prime}}^{\ell} \bar{\ell} \gamma^{\mu} \ell Z_{\mu}^{\prime}
$$

\subsection{The "pseudo-observables" approach}

The PO formalism described here is based on the specification of the pole structure of the amplitude for the entire $H \rightarrow 4 \ell$ process. As we are considering an on-shell Higgs, and we single out the pole terms due to the exchange of the SM gauge bosons, the resulting

\footnotetext{
${ }^{5} \mathrm{An}$ alternate and perhaps clearer approach would be to integrate out the heavy $Z^{\prime}$. We have chosen the approach above, with explicit $\kappa_{1, Z Z^{\prime}}$ term in the interest of consistency with parameterizations already in use by the experiments.
} 
amplitude is automatically gauge invariant, which is an advantage with respect to the "amplitude" approach described in subsection 2.1, especially when considering the effects of higher order corrections [160]. ${ }^{6}$ Furthermore, the PO formalism, like the amplitude formalism, can accommodate arbitrary loop corrections in a relatively straightforward way.

Following ref. [156], the goal of $H \rightarrow 4 \ell$ studies in the PO approach is to characterize in general terms the correlation function of the Higgs and two fermion currents,

$$
\left\langle 0\left|\mathcal{T}\left\{H(0), J_{\ell}^{\mu}(x), J_{\ell^{\prime}}^{\nu}(y)\right\}\right| 0\right\rangle,
$$

where $\ell, \ell^{\prime}=e, \mu$. Assuming Lorentz invariance and reasonable assumptions about flavor conservation, the matrix element corresponding to this correlation function can be described by

$$
\begin{aligned}
& \mathcal{A}_{n . c .}\left[X \rightarrow \ell^{-}\left(p_{1}\right) \ell^{+}\left(p_{2}\right), \ell^{\prime-}\left(p_{3}\right) \ell^{\prime+}\left(p_{4}\right)\right]=i \frac{2 m_{Z}^{2}}{v} \sum_{\ell=\ell_{L}, \ell_{R}} \sum_{\ell^{\prime}=\ell_{L}^{\prime}, \ell_{R}^{\prime}}\left(\bar{\ell} \gamma_{\rho} \ell\right)\left(\bar{\ell}^{\prime} \gamma_{\sigma} \ell^{\prime}\right) \mathcal{T}^{\rho \sigma}\left(q_{1}, q_{2}\right) \\
& \mathcal{T}^{\rho \sigma}\left(q_{1}, q_{2}\right)=[ F_{1}^{\ell \ell^{\prime}}\left(q_{1}^{2}, q_{2}^{2}\right) g^{\rho \sigma}+F_{3}^{\ell \ell^{\prime}}\left(q_{1}^{2}, q_{2}^{2}\right) \frac{q_{1} \cdot q_{2} g^{\rho \sigma}-q_{2}^{\rho} q_{1}^{\sigma}}{m_{Z}^{2}} \\
&\left.+F_{4}^{\ell \ell^{\prime}}\left(q_{1}^{2}, q_{2}^{2}\right) \frac{\varepsilon^{\rho \sigma \alpha \beta} q_{V_{2} \alpha} q_{V_{1}} \beta}{m_{Z}^{2}}\right],
\end{aligned}
$$

where $q_{1}=p_{1}+p_{2}$ and $q_{2}=p_{3}+p_{4}$. Recognizing the presence of physical poles in the correlation function (2.8) due to the propagation of intermediate SM gauge bosons, we expand around these poles and define the PO directly from their residues:

$$
\begin{aligned}
F_{1}^{\ell \ell^{\prime}}\left(q_{1}^{2}, q_{2}^{2}\right)= & \kappa_{Z Z} \frac{g_{Z}^{\ell} g_{Z}^{\ell^{\prime}}}{P_{Z}\left(q_{1}^{2}\right) P_{Z}\left(q_{2}^{2}\right)}+\frac{\epsilon_{Z \ell}}{m_{Z}^{2}} \frac{g_{Z}^{\ell^{\prime}}}{P_{Z}\left(q_{2}^{2}\right)}+\frac{\epsilon_{Z \ell^{\prime}}}{m_{Z}^{2}} \frac{g_{Z}^{\ell}}{P_{Z}\left(q_{1}^{2}\right)} \\
& +\Delta_{1}^{\mathrm{SM}}\left(q_{1}^{2}, q_{2}^{2}\right), \\
F_{3}^{\ell \ell^{\prime}}\left(q_{1}^{2}, q_{2}^{2}\right)= & \epsilon_{Z Z} \frac{g_{Z}^{\ell} g_{Z}^{\ell^{\prime}}}{P_{Z}\left(q_{1}^{2}\right) P_{Z}\left(q_{2}^{2}\right)}-\epsilon_{Z \gamma}\left(\frac{e g_{Z}^{\ell}}{q_{2}^{2} P_{Z}\left(q_{1}^{2}\right)}+\frac{e g_{Z}^{\ell^{\prime}}}{q_{1}^{2} P_{Z}\left(q_{2}^{2}\right)}\right) \\
& +\frac{\epsilon_{\gamma \gamma} e^{2}}{q_{1}^{2} q_{2}^{2}}+\Delta_{3}^{\mathrm{SM}}\left(q_{1}^{2}, q_{2}^{2}\right), \\
F_{4}^{\ell \ell^{\prime}}\left(q_{1}^{2}, q_{2}^{2}\right)= & \epsilon_{Z Z}^{\mathrm{CP}} \frac{g_{Z}^{\ell} g_{Z}^{\ell^{\prime}}}{P_{Z}\left(q_{1}^{2}\right) P_{Z}\left(q_{2}^{2}\right)}-\epsilon_{Z \gamma}^{\mathrm{CP}}\left(\frac{e g_{Z}^{\ell}}{q_{2}^{2} P_{Z}\left(q_{1}^{2}\right)}+\frac{e g_{Z}^{\ell^{\prime}}}{q_{1}^{2} P_{Z}\left(q_{2}^{2}\right)}\right) \\
& +\frac{\epsilon_{\gamma \gamma}^{\mathrm{CP}} e^{2}}{q_{1}^{2} q_{2}^{2}},
\end{aligned}
$$

where $g_{Z}^{\ell, \ell^{\prime}}$ correspond to the well-measured LEP-I $Z$-pole PO,

$$
\mathcal{A}\left(Z(\varepsilon) \rightarrow \ell^{+} \ell^{-}\right)=i \sum_{\ell=\ell_{L}, \ell_{R}} g_{Z}^{\ell} \varepsilon_{\nu} \bar{\ell} \gamma^{\nu} \ell
$$

\footnotetext{
${ }^{6}$ If we were to consider contributions to high invariant mass four-lepton events from an off-shell Higgs boson [69, 88, 95, 99, 164-179], we would need to construct PO for the whole $g g \rightarrow 4 \ell$ process, including both the "signal" $g g \rightarrow H \rightarrow V V \rightarrow 4 \ell$ amplitude and the "background" $g g \rightarrow V V \rightarrow 4 \ell$ amplitudes with which it interferes.
} 


\begin{tabular}{|c|c|c|}
\hline PO & Lagrangian parameter & Amplitude parameters \\
\hline$\kappa_{Z Z}$ & $-\kappa_{1, Z Z}-\kappa_{4, Z Z}-2 \kappa_{5, Z Z}$ & $\frac{1}{2} a_{1}^{Z Z}+\frac{m_{Z}^{2}}{\left(\Lambda_{1}^{Z Z}\right)^{2}} \kappa_{1}^{Z Z}$ \\
$\epsilon_{Z Z}$ & $\kappa_{2, Z Z}$ & $a_{2}^{Z Z}$ \\
$\epsilon_{Z Z}^{\mathrm{CP}}$ & $\kappa_{3, Z Z}$ & $a_{3}^{Z Z}$ \\
$\epsilon_{Z \gamma}$ & $\kappa_{2, Z \gamma} / 2$ & $a_{2}^{Z \gamma}$ \\
$\epsilon_{Z \gamma}^{\mathrm{CP}}$ & $\kappa_{3, Z \gamma} / 2$ & $a_{3}^{Z \gamma}$ \\
$\epsilon_{\gamma \gamma}$ & $\kappa_{2, \gamma \gamma}$ & $a_{2}^{\gamma \gamma}$ \\
$\epsilon_{\gamma \gamma}^{\mathrm{CP}}$ & $\kappa_{3, \gamma \gamma}$ & $a_{3}^{\gamma \gamma}$ \\
$\epsilon_{Z f}$ & $-g_{Z}^{f} \kappa_{5, Z Z}+g_{Z^{\prime}}^{f} \frac{\kappa_{1, Z Z^{\prime}}}{2} \frac{m_{Z}^{2}}{m_{Z^{\prime}}^{2}}-e \frac{\kappa_{5, Z \gamma}}{2} \frac{m_{Z}^{2}}{m_{H}^{2}}$ & $g_{Z}^{f} \frac{\kappa_{1}^{Z Z} m_{Z}^{2}}{2\left(\Lambda_{1}^{Z Z}\right)^{2}}-g_{Z^{\prime}}^{f} \frac{a_{1}^{Z Z^{\prime}} m_{Z}^{2}}{2 m_{Z^{\prime}}^{2}}-e \frac{\kappa_{2}^{Z \gamma} m_{Z}^{2}}{2\left(\Lambda_{1}^{Z \gamma}\right)^{2}}$ \\
\hline
\end{tabular}

Table 1. In this table we provide a "dictionary", allowing one to convert (at the tree level) between (i) the "amplitude" formalism of eq. (2.1), (ii) the "Lagrangian" formalism of eq. (2.3), and (iii) the "pseudo-observables" formalism of eq. (2.9). The label $f$ is a generic label for a fermion $\left(f=e_{L}\right.$, $\left.e_{R}, \mu_{L}, \mu_{R}\right)$.

and $P_{Z}\left(q^{2}\right)=q^{2}-m_{Z}^{2}+i m_{Z} \Gamma_{Z}$. Note the minus signs in eqs. (2.11) and (2.12) occur because the relevant expressions contain a $Q$ for the fermion charge in ref. [156], which is equal to minus one for electrons and muons. In this decomposition around physical poles, we neglected further terms which are necessarily generated by local operators of dimension greater than 6 and thus strongly suppressed if the new physics scale is above the electroweak scale. The parameters $g_{Z}^{f}, \kappa_{Z Z}$, and $\epsilon_{X}$ are thus well-defined PO; they can be measured in experiments and their values can be calculated at any given order in perturbation theory in any underlying theory (or effective field theory). ${ }^{7}$

While the amplitude for $H \rightarrow 2 e 2 \mu$ is given directly by eq. (2.9), the decays $H \rightarrow$ $4 e, 4 \mu$ include an interference between two contributions, corresponding to the two possible assignments of the fermions inside the currents:

$$
\begin{aligned}
\mathcal{A}\left[h \rightarrow \ell\left(p_{1}\right) \bar{\ell}\left(p_{2}\right) \ell\left(p_{3}\right) \bar{\ell}\left(p_{4}\right)\right]= & \mathcal{A}_{n . c .}\left[h \rightarrow f\left(p_{1}\right) \bar{f}\left(p_{2}\right) f^{\prime}\left(p_{3}\right) \bar{f}^{\prime}\left(p_{4}\right)\right]_{f=f^{\prime}=\ell} \\
& -\mathcal{A}_{n . c .}\left[h \rightarrow f\left(p_{1}\right) \bar{f}\left(p_{4}\right) f^{\prime}\left(p_{3}\right) \bar{f}^{\prime}\left(p_{2}\right)\right]_{f=f^{\prime}=\ell}
\end{aligned}
$$

The tree-level connection between the PO and the parameters used in eqs. (2.1) and (2.3) is given in table 1. Of these parameters, only $\epsilon_{Z e_{L, R}}$ and $\epsilon_{Z \mu_{L, R}}$ can depend on the flavor of the final-state leptons. In the limit, where we neglect loop contributions from light states, $\kappa_{Z Z}$ and $\epsilon_{X}$ are all real. The functions $\Delta_{1,3}^{\mathrm{SM}}\left(q_{1}^{2}, q_{2}^{2}\right)$ describe loop-induced SM contributions, which cannot be described in terms of $D \leq 6$ effective operators; an explicit expression for $\Delta_{3}^{\mathrm{SM}}\left(q_{1}^{2}, q_{2}^{2}\right)$ at one-loop can be found in ref. [156], where it is shown that their size is small and thus can be neglected given experimental precision in the foreseeable future.

The tree-level matching of the operators in the Lagrangian approach to the PO, which is shown in table 1 , is obtained by computing the same decay amplitude and rearranging the terms so that the expansion around physical poles is recovered. For example, the

\footnotetext{
${ }^{7}$ Here we generically denote by $\epsilon_{X}$ the parameters $\epsilon_{Z Z, Z \gamma, \gamma \gamma, Z f}$ and $\epsilon_{Z Z, Z \gamma, \gamma \gamma}^{\mathrm{CP}}$.
} 
$H Z_{\mu} \partial_{\nu} F^{\mu \nu}$ operator in eq. (2.3) corresponds to an amplitude with a $q_{\gamma}^{2}$ in the numerator (from the $\square$ operator). This $q_{\gamma}^{2}$ cancels the photon propagator in the denominator, so this operator contributes to $\epsilon_{Z e}$ and $\epsilon_{Z \mu}$. The contribution of the heavy $Z^{\prime}$ is evaluated in the limit $q_{V}^{2} \ll m_{Z^{\prime}}^{2}$ (consistently with our general assumptions). In such limit the $Z^{\prime}$ can easily be integrated out, generating a contribution to the contact terms with the flavor structure given by its couplings to fermions. Let us stress, however, that the $Z^{\prime}$ is just a specific example of a BSM scenario that generates flavor-dependent contact terms. The PO approach is not restricted in any sense to this particular case, in contrast to the Amplitude or Lagrangian approaches.

\subsection{Global approaches to Higgs couplings}

While our interest in this paper is in detailed parameterizations of BSM effects in $H \rightarrow 4 \ell$, we note in passing that much work has gone into "global" studies of Higgs couplings to various final states, whether in the so-called " $\kappa$ " or "signal-strength" formalism [180-187]) or in the context of SM effective theories [80, 167, 172, 188-249]. While such studies are clearly of value, we wish to emphasize that our goal here is quite different. Specifically, our goal is to allow a description of BSM effects in $H \rightarrow 4 \ell$ that is as theoretically unbiased as possible using as much information as possible.

Generally, more global approaches to Higgs couplings make numerous additional assumptions and use less information about a specific process, such as Higgs to four leptons, than is experimentally available. As the assumptions in these studies tend to be theoretically reasonable, such work is extremely useful in providing a "big picture" view of what many different channels are telling us about the Higgs, which is complementary to our goal of extracting the most possible information from a single channel. To give an indication of what is possible in such analyses and to illustrate the use of the various formalisms described here, particularly the PO framework presented in subsection 2.3, we present projections, in section 3, for the sensitivity of LHC measurements in these frameworks.

\section{LHC projections}

\subsection{Benchmark scenarios}

While the $\mathrm{PO}$ are directly related to physical properties of the Higgs decay amplitudes, the generic expectation is that explicit new physics models would contribute to some combination of PO. For this reason, the experimental analysis should be performed with the most general case possible in mind. In order to reduce the number of independent parameters, the best option is to impose relations due to specific symmetries [156].

With this important caveat in mind, but also with an eye to studying the sensitivity of the LHC and other future colliders for measuring Higgs PO in $H \rightarrow 4 \ell$ decays, let us list the following six simplified scenarios, each of which has, conveniently, only two independent PO:

1. Longitudinal vs. transverse: $\left(\kappa_{Z Z}\right.$ vs. $\left.\epsilon_{Z Z}\right)$, where all other $\epsilon \rightarrow 0$.

2. CP admixture: ( $\kappa_{Z Z}$ vs. $\left.\epsilon_{Z Z}^{\mathrm{CP}}\right)$, where all other $\epsilon \rightarrow 0$. 
3. Linear EFT-inspired [114]: ( $\kappa_{Z Z}$ vs. $\left.\epsilon_{Z \ell_{R}}\right)$, where $\epsilon_{Z \ell_{L}}=2 \epsilon_{Z \ell_{R}}, \epsilon_{Z e_{L, R}}=\epsilon_{Z \mu_{L, R}}$, and other $\epsilon \rightarrow 0$ (or $\kappa_{Z Z}$ vs. some other combination of contact terms).

4. Flavor universal contact terms: $\left(\epsilon_{Z \ell_{L}}\right.$ vs. $\left.\epsilon_{Z \ell_{R}}\right)$, where $\epsilon_{Z e_{L, R}}=\epsilon_{Z \mu_{L, R}}, \kappa_{Z Z}=1$, and other $\epsilon \rightarrow 0$.

5. Flavor non-universal vector contact terms: $\left(\epsilon_{Z e_{R}}\right.$ vs. $\left.\epsilon_{Z \mu_{R}}\right)$, where $\epsilon_{Z e_{L}}=\epsilon_{Z e_{R}}$, $\epsilon_{Z \mu_{L}}=\epsilon_{Z \mu_{R}}, \kappa_{Z Z}=1$, and other $\epsilon \rightarrow 0$.

6. Flavor non-universal axial contact terms: $\left(\epsilon_{Z e_{R}}\right.$ vs. $\left.\epsilon_{Z \mu_{R}}\right)$, where $\epsilon_{\ell_{L}}=-\epsilon_{Z \ell_{R}}$, $\kappa_{Z Z}=1$, and other $\epsilon \rightarrow 0$.

Scenarios 1 and 2 have been the focus of much of the existing work in four-lepton phenomenology and experiment, whereas the rest of cases have received much less attention. Scenarios 1, 2, and 3 include $\kappa_{Z Z}$, which only affects the overall normalization of all $H \rightarrow 4 \ell$ channels and which consequently can only be probed through its effect in the total rates. This in turn requires the implicit assumption that other BSM effects affecting Higgs production are absent. For this reason in the next subsections we focus on the last three scenarios, where kinematic distributions and ratios of rates also provide interesting information on all PO under study. This study will serve as an example of the use of the PO formalism. These scenarios all involve contact operators that couple the Higgs to an intermediate boson and two leptons. They could arise, e.g., via a heavy $Z^{\prime}$ that is integrated out, but also via other mechanisms that might not even involve two intermediate bosons. In scenario 4, this interaction is of the same strength for electrons and muons; the helicity structure of the coupling gives us two independent parameters. In scenarios 5 and 6 , we fix the helicity structure of the couplings to be vector or axial, respectively, and make the couplings to electrons and muons the two independent parameters. The violation of lepton-flavor universality makes these two scenarios qualitatively different from the rest, both conceptually and in practice, as we will see in the phenomenological analyses below.

\subsection{Projections using rates and kinematics}

The ATLAS collaboration has recently published the first $h \rightarrow 4 \ell$ analysis in the PO framework using $36.1 \mathrm{fb}^{-1}$ of data at $13 \mathrm{TeV}$ [250] (see also [251]). They work in the (lepton flavor universal) benchmark scenarios 3 and 4 , using the binned $\left(m_{12}, m_{34}\right)$ invariant mass distribution as experimental input. Let us stress that they do not work with normalized distributions, and thus they are not only sensitive to the effects of the PO in the shapes but also in the total rates. As mentioned above, this assumes SM-like Higgs production, which allows them to probe $\kappa_{Z Z}$ in scenario 3 .

In this section we expand the scope of this ATLAS search and go one step beyond, highlighting the importance of lepton flavor universality tests. Namely, we split the $\left(m_{12}, m_{34}\right)$ histogram, shown in figure 3 (right) of [250], into four categories based on the lepton flavor: $4 e, 4 \mu, 2 e 2 \mu$, and $2 \mu 2 e .^{8}$ Apart from that, we closely follow the ATLAS analysis of ref. [250] and obtain projections for our scenarios 4,5 and 6 .

\footnotetext{
${ }^{8}$ The channels $2 e 2 \mu$ and $2 \mu 2 e$ are split according to which lepton pair comes from the onshell $\mathrm{Z}$ boson, or more precisely, which one has an invariant mass closest to the $Z$ mass.
} 

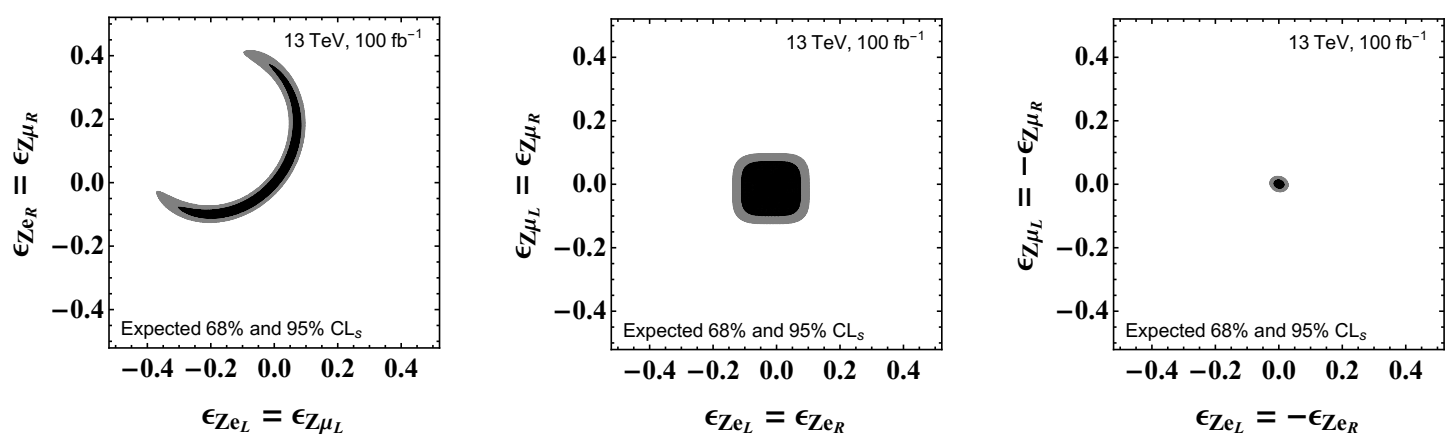

Figure 1. Projection of the ATLAS analysis $[250]$ to $100 \mathrm{fb}^{-1}(\sqrt{s}=13 \mathrm{TeV})$ for scenarios 4,5 , and 6. Black (gray) regions represent 68\% (95\%) expected CL intervals.

Let us first explain our simulation procedure. The signal events $(p p \rightarrow h \rightarrow 4 \ell)$ are generated using the HiggsPO UFO model from ref. [163] within the MadGraph5_aMC@NLO framework [255]. Reliable gluon-gluon fusion production kinematics is obtained with the leading order matrix element and parton shower jet merging, while the normalization factor $\left(K_{F}=2.32\right)$ is taken from the best higher-order QCD prediction [252]. Subsequent showering and hadronisation effects are simulated with Pythia 6 [253], while the detector effects are simulated with Delphes 3 [254]. Event samples are generated for enough points in the PO parameter space allowing for the reconstruction of the quadratic dependence of any observable (to be discussed below). The dominant background, coming from $p p \rightarrow Z Z^{*}\left(\gamma^{*}\right) \rightarrow 4 \ell$, follows a similar simulation pipeline. We estimate the NLO QCD effects for the background by computing the $K$-factor in the signal region $\left(K_{F}=1.3\right)$, and using it to rescale the LO simulation.

We closely follow the event selection of the ATLAS search. The signal region is defined as follows. Four leptons $(\ell=e, \mu)$ are selected to make a lepton quadruplet. Electrons are required to have $E_{T}>7 \mathrm{GeV}$ and $\eta<2.47$, while muons satisfy $p_{T}>5 \mathrm{GeV}$ and $\eta<2.7$. Jets are reconstructed with the anti- $k_{T}$ algorithm and considered when $p_{T}>30 \mathrm{GeV}$ and $\eta<4.5$. A lepton quadruplet consisting of two pairs of same flavor opposite-charge leptons is required, with the $p_{T}$ cuts in the quadruplet of $20 \mathrm{GeV}, 15 \mathrm{GeV}$ and $10 \mathrm{GeV}$ for the leading leptons. Quadruplets with same flavor opposite-charge lepton's invariant mass below $5 \mathrm{GeV}$ are discarded. The opposite sign same flavor lepton pair closest to the $Z$-boson mass is referred as the leading dilepton, with invariant mass, $m_{12}$, required to be between $50 \mathrm{GeV}$ and $106 \mathrm{GeV}$. The sub-leading dilepton invariant mass, $m_{34}$, is required to be in the range $12 \mathrm{GeV}$ to $115 \mathrm{GeV}$. As a validation of our recast procedure, we correctly reproduce the ATLAS expected signal and background events in figure 3 (right) of ref. [250].

The number of events obtained in the each bin is proportional to the corresponding squared matrix element, which is a quadratic function of the PO. Therefore, we introduce matrices, $X_{i j}$, for every bin to express the number of events as

$$
\frac{N}{N^{S M}}=\sum_{j \geq i} X_{i j} \epsilon_{i} \epsilon_{j}
$$


where $N^{S M}$ is the number of expected events in the SM and where we introduced the vector $\epsilon \equiv\left(\epsilon_{Z e_{L}}, \epsilon_{Z e_{R}}, \epsilon_{Z \mu_{L}}, \epsilon_{Z \mu_{R}}\right)^{T}$. The latter only contains contact terms because we focus on the benchmark scenarios 4,5 and 6 . The extension to a more general case with more PO is straightforward. In order to determine the $X$ matrices, we need to run the simulation for different values of the Higgs PO as the input parameters. This needs to be done ten times at least, since this is the number of independent parameters of a real, symmetric, $4 \times 4$ matrix. Furthermore, we divided the points in the $m_{12}-m_{34}$ plane into five bins, as was done in the ATLAS search [250]. After we calculate the $X$ matrices, we can compare "exotic PO" with the "SM Higgs" by constructing the appropriate likelihood function. Although we will work with a fix luminosity of $100 \mathrm{fb}^{-1}$, let us note that the $X_{i j}$ coefficients do not scale with the luminosity.

The events are categorised by four decay channels, $h \rightarrow 4 e, h \rightarrow 4 \mu, h \rightarrow 2 e 2 \mu$ and $h \rightarrow 2 \mu 2 e$, each further separated into five bins. The likelihood function is given by the product of Poisson probabilities for all bins and all categories:

$$
L(\epsilon)=\prod_{n} \frac{\exp \left(-\mu_{n}\right)\left(\mu_{n}\right)^{N_{n}^{\exp }}}{N_{n}^{\exp } !},
$$

where the index $n$ refers to a specific bin and category, and

$$
\mu_{n}=N_{n}^{\mathrm{bkg}, \mathrm{SM}}+N_{n}^{\mathrm{sig}, \mathrm{SM}} \epsilon^{T} X_{n} \epsilon .
$$

The quantities $N_{n}^{\text {bkg,SM }}$ and $N_{n}^{\text {sig,SM }}$ represent the number of events per category and bin for background and signal events within the SM, respectively. $N_{n}^{\exp }$ is number of events per bin and category obtained in the experiment. For the purpose of this study, we neglect systematic uncertainties.

Using the likelihood function in eq. (3.2), we construct a profile likelihood ratio statistic, $\lambda(\epsilon) \equiv-2 \log (L(\epsilon) / L(\hat{\epsilon}))$, to obtain $68 \%$ and $95 \%$ expected confidence levels for $100 \mathrm{fb}^{-1}$ assuming the SM. Our results for scenarios 4, 5 and 6 , are shown in figure 1 from left to right, respectively. In the following, we will try to break down these limits and understand the impact of the overall normalization, lepton universality rate ratios and event kinematics separately.

\subsection{Projections using relative normalization and kinematics}

In this section, we study again the sensitivity of future LHC data to Higgs pseudoobservables using benchmark scenarios 4-6. However, in contrast to the analysis in the previous subsection, we will not include the information contained in the overall normalization common to all $H \rightarrow 4 \ell$ flavor channels. Moreover, we will not use the binned $\left(m_{12}, m_{34}\right)$ invariant mass distribution but will instead use the unbinned one with full event-by-event information. This approach is similar to that followed in, e.g., the CMS search described in ref. [22].

In addition to presenting results derived using only kinematic information, we will also present results where the discovery significance shown results from both kinematical information and "relative normalization", i.e., the relative number of $4 e, 4 \mu$, and $2 e 2 \mu$ 
events. These analyses help us to understand how much of the sensitivity to exotic PO points is due to kinematical information, how much is due to changes in the ratio of various event types, and how much is due to the overall normalization.

To ease the comparison with the results of the previous subsection we work again with a fixed luminosity of $100 \mathrm{fb}^{-1}$ at $13 \mathrm{TeV}$. Figures 2-4 show discovery projections for the non-standard contact terms, $\epsilon_{Z \ell}$, present in scenarios 4-6. These projections are obtained using the Matrix Element Method (MEM) [256-264], in essentially the same manner as in ref. [92]. Let us discuss below the technical details.

Since the likelihood ratio, the essential quantity to calculate in the MEM, reduces to the ratio of squared matrix elements between different coupling hypotheses, our analysis is built on such "discriminants", specifically

$$
\mathcal{D}_{\mathrm{SM}}=\ln \frac{\left|\mathcal{M}_{\mathrm{H}}\left(p \mid m_{H}=M\right)\right|^{2}}{\left|\mathcal{M}_{\mathrm{ZZ}}\right|^{2}},
$$

and

$$
\mathcal{D}_{\text {exo }}=\ln \frac{\left|\mathcal{M}_{\mathrm{H}}\left(p \mid m_{H}=M\right)\right|^{2}}{\left|\mathcal{M}_{\text {exo }}\left(p \mid m_{\text {exo }}=M\right)\right|^{2}},
$$

where $M$ is the invariant mass of the four lepton system, $\left|\mathcal{M}_{\mathrm{H}}\left(p \mid m_{H}=M\right)\right|^{2}$ is the squared matrix element for the four-lepton momenta, $p$, under the hypothesis that the four leptons are produced by the decay of an SM Higgs with mass $M$ (and only tree level couplings), $\left|\mathcal{M}_{\text {exo }}\left(p \mid m_{\text {exo }}=M\right)\right|^{2}$ is the squared matrix element for the four-lepton momenta, $p$, under the hypothesis that the four leptons are produced by the decay of a Higgs boson with the exotic (non-SM) PO indicated by the axes of the corresponding plot, and $\left|\mathcal{M}_{\mathrm{ZZ}}\right|^{2}$ is the squared matrix element for the four-lepton momenta $p$ under the hypothesis that the four leptons are produced by (leading order) SM $q \bar{q} \rightarrow 4 \ell$ processes.

To perform the analysis, we obtain the 2-D histograms ("templates" or, in statistical language, probability mass functions) in dimensions of $\mathcal{D}_{\mathrm{SM}}$ and $\mathcal{D}_{\text {exo }}$ using parton-level signal and background events. The SM normalization per template is applied as is appropriate, which is derived using the SM $H \rightarrow 4 \ell$ and $q \bar{q} \rightarrow 4 \ell$ processes. ${ }^{9}$ The parton-level histograms were obtained using a background sample of 140 thousand SM $q \bar{q} \rightarrow 4 \ell$ events and a signal sample which, after selection cuts, consists of 560 thousand SM $H \rightarrow 4 \ell$ events. The SM Higgs and $q \bar{q} \rightarrow 4 \ell$ events were obtained using MG5_aMC@NLO [255]; to obtain the vast set of samples that model all the $\gtrsim 150$ coupling points, a matrix-element-based event reweighting was applied, thus enabling a creation of exotic signal templates from the SM Higgs events (see, e.g., ref. [265]). We conservatively account for mass resolution effects, as in ref. [92], by including all events within a "window" of $6 \mathrm{GeV}$ around the Higgs boson mass, which we take to be $125 \mathrm{GeV}$. Separate histograms were produced for each four-lepton final-state flavor, i.e., $4 e, 4 \mu$, and $2 e 2 \mu$. This allows us both to consider the effects of interference, which arise between the amplitudes for different lepton pairings in the $4 e$ and $4 \mu$ final states (see, e.g., ref. [79]), and to consider the effects of flavor non-universal anomalous $\mathrm{PO}$ in scenarios 5 and 6.

\footnotetext{
${ }^{9}$ As mentioned above, we assume throughout this analysis that the exotic coupling point has the same cross section as the SM, i.e., we do not use the overall normalization in our analysis.
} 
As the $\mathcal{D}_{\text {exo }}$ discriminant depends on the chosen coupling point, we need to obtain templates for the three final states, each of different lepton flavor, and for the three event types "SM Higgs", "exotic PO", and "background", for all $7 \times 7$ coupling points that we evaluate for each scenario: a total of $3 \times 3 \times 7 \times 7=441$ templates. For scenario 4 , we construct templates at additional coupling points to make the plot of the expanded ("zoomed-in") region (figure $2 \mathrm{~b}$ ). Having the necessary templates, we (a) produce the distributions of $\log$-likelihood ${ }^{10}$ values for 50000 4-lepton pseudo-experiment events per coupling point, with the number of expected signal and background events given by the SM Higgs and background event yields, respectively, from ref. [92] and (b) produce the distributions of log-likelihood values for 50000 4-lepton pseudo-experiment events per coupling point, with the number of expected $4 e, 4 \mu$, and $2 e 2 \mu$ events in the ratio predicted for the PO point, but with the total number of $4 e, 4 \mu$, and $2 e 2 \mu$ events set to the SM value.

Our immediate aim, for each exotic PO point, and for each of these two analyses, was to determine the mean separation power between the "SM Higgs" and the "exotic PO". This is done by evaluating the distance in $\sigma_{S M}$ between the means of corresponding loglikelihood distributions. Such a separation criterion can be loosely thought of as a mean $\mathrm{p}$ value and is defined here as the following:

$$
\mathrm{p} \text { value }=\frac{\langle\text { exotic } \mathrm{PO}\rangle-\langle\text { SM Higgs }\rangle}{\sigma_{S M}},
$$

where $\langle$ exotic $\mathrm{PO}\rangle$ ( $\langle\mathrm{SM}$ Higgs $\rangle$ ) is the mean of the log-likelihood distribution when the exotic coupling point (the SM Higgs) is the true hypothesis. To be conservative, we included 5\% normalization (yield) uncertainties for each of the several different (independent) sources that are typical to experiments, namely (i) the total event count due to theoretical knowledge, (ii) the total event count due to partonic density mismodeling, (iii) the total event count due to generic detector inefficiencies, (iv) the expected number of exotic PO events, (v) the expected number of the SM Higgs events, and (vi) the expected number of background events. These uncertainties effectively smear (widen) the log-likelihood distributions, thus increasing the $\sigma_{S M}$ denominator in eq. (3.6). In the limit of large numbers of expected events, as for the $100 \mathrm{fb}^{-1}$ considered in figures $2-4$, this quantity corresponds to the $Z$ value $^{11}$ of the expected discovery significance and can be scaled in a straightforward way to obtain predictions for other luminosities. The results of the analyses of type (a) are shown in the left panels of figures $2-4$ and depend on only kinematical properties of the events, while the results of analyses of type (b) are shown in the right panels figures 2-4 and depend on both kinematical information and the relative number of events in each flavor channel that one would obtain from various PO points.

\subsection{Discussion of results}

The comparison of the results obtained using rates and kinematical information with the results obtained using only kinematical information in our benchmark scenarios shows that

\footnotetext{
${ }^{10}$ The probability mass functions, which are the above-described templates, times the Poisson probability density functions are used to evaluate the likelihoods.

${ }^{11}$ By " $Z$ value" we mean, essentially, the number of " $\sigma$ " describing the statistical significance of a result.
} 


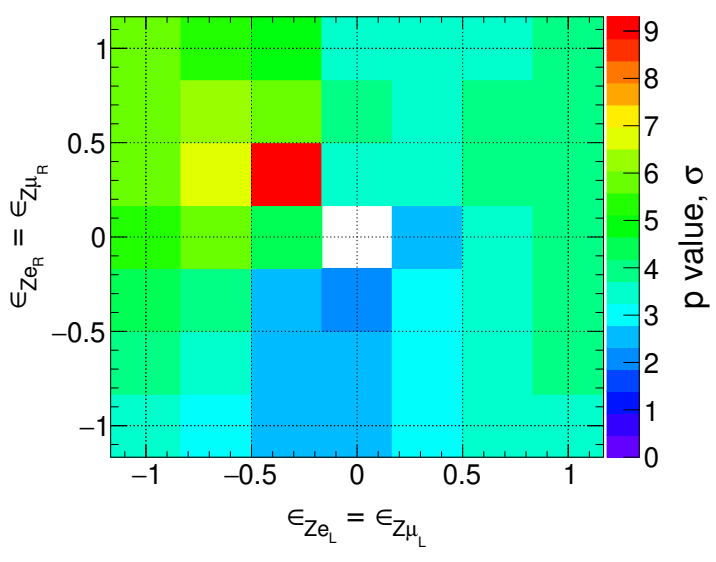

(a) A full-range version.

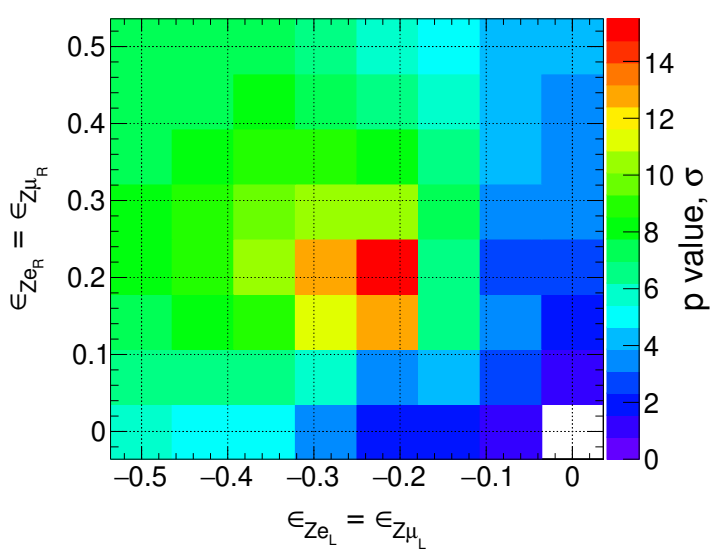

(b) A zoomed-in version.

Figure 2. These figures show the expected discovery sensitivity in sigma (with $100 \mathrm{fb}^{-1}$ at $13 \mathrm{TeV}$ ) for the hypothesis described in scenario 4, where, in addition to the SM coupling, the PO $\epsilon_{Z \ell_{L}}=$ $\epsilon_{Z e_{L}}=\epsilon_{Z \mu_{L}}$ and $\epsilon_{Z \ell_{R}}=\epsilon_{Z e_{R}}=\epsilon_{Z \mu_{R}}$ take on non-zero values. These quantities are specified on the $\mathrm{x}$ and $\mathrm{y}$ axes; the $\mathrm{SM}$ is reflected by the point $(0,0)$. The figure on the right, figure $2 \mathrm{~b}$, gives a zoomed-in version of the second quadrant of figure $2 \mathrm{a}$. The results are obtained using only the kinematical distribution of the events via the procedure specified in subsection 3.3. Including information about the relative normalization of the various $H \rightarrow 4 \ell$ channels has a relatively small impact here.

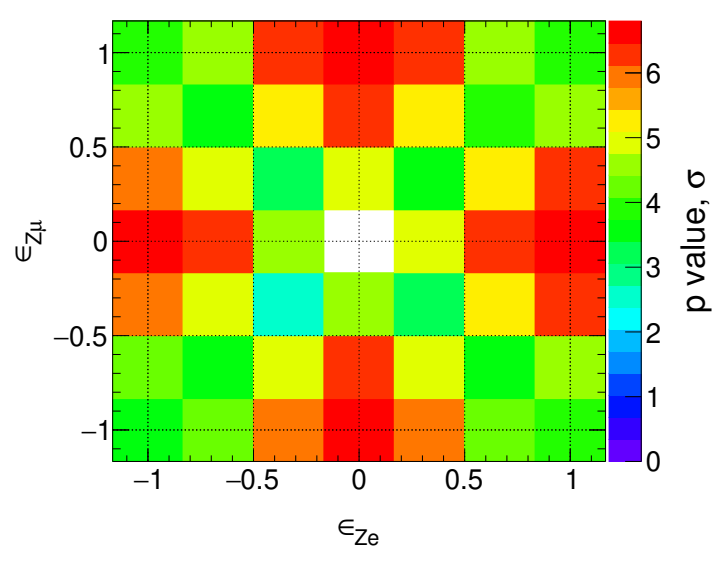

(a) Kinematics.

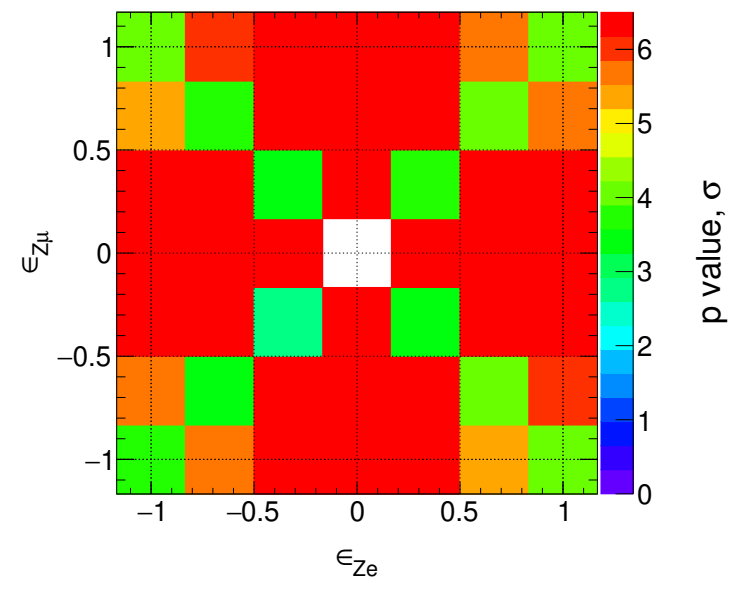

(b) Kinematics and relative normalization.

Figure 3. The left figure is the same as figure 2a, except that in this case we are considering the benchmark scenario 5, in which the anomalous $\mathrm{PO}$ are $\epsilon_{Z e}=\epsilon_{Z e_{L}}=\epsilon_{Z e_{R}}$ and $\epsilon_{Z \mu}=\epsilon_{Z \mu_{L}}=\epsilon_{Z \mu_{R}}$. The right figure is obtained also for scenario 5 but including not only kinematics but also information about the relative normalization of the various $H \rightarrow 4 \ell$ flavor channels. 


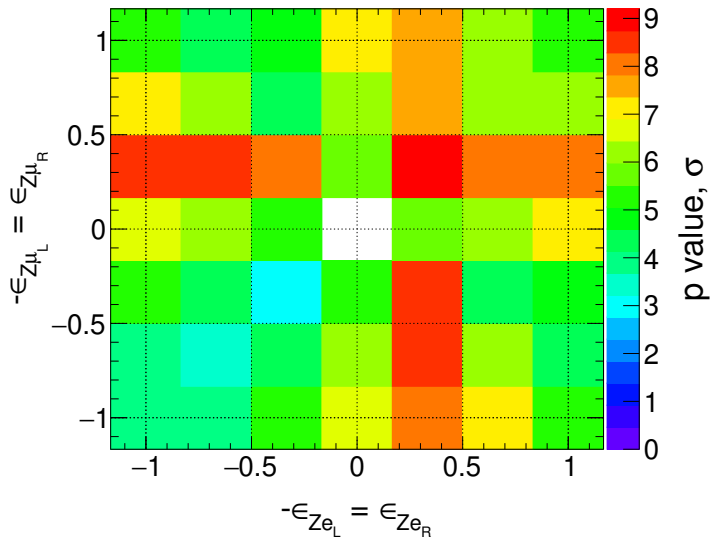

(a) Kinematics.

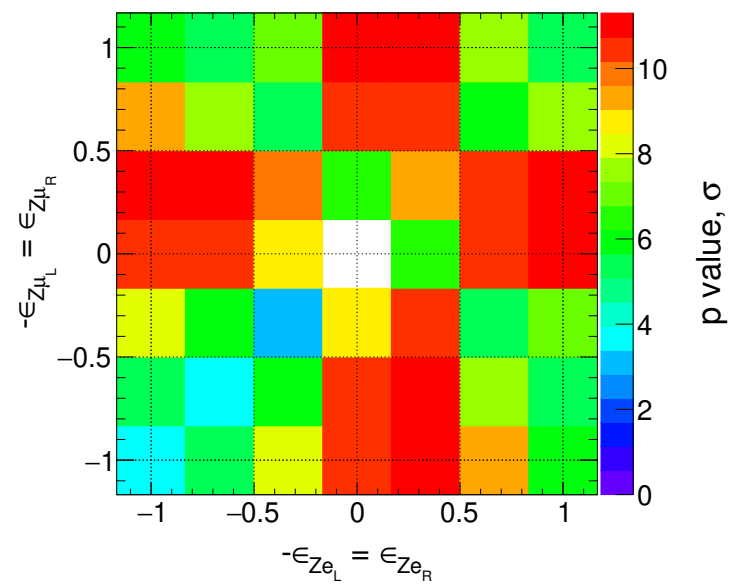

(b) Kinematics and relative normalization.

Figure 4. This figure is the same as figure 2a, except that here we are considering scenario 6 , in which the anomalous $\mathrm{PO}$ are $\epsilon_{Z e_{R}}=-\epsilon_{Z e_{L}}$ and $\epsilon_{Z \mu_{R}}=-\epsilon_{Z \mu_{L}}$.

the overall normalization of all $H \rightarrow 4 \ell$ channels provides by far the main sensitivity to the contact terms. However it is important to note that kinematic distributions and ratios of rates do provide additional information that allows one to exclude regions of parameter space with reasonably large nonstandard terms that do not affect the overall normalization much due to accidental cancellations. This is why we do not find a perfect ring-shape region in figure 1 for scenario 4 (the impact on scenario 5 and 6 is actually much larger).

As mentioned before, the large sensitivity of the overall normalization to contact terms comes however at a price: the implicit assumption that other BSM effects affecting Higgs production (or the SM-like pseudo-observable $\kappa_{Z Z}$ ) are absent. On the other hand, the relative normalization or the kinematic distribution of the events do not require this assumption. Our results in figures $2-4$ show that these normalized observables provide a significant sensitivity to the contact terms in a large part of the parameter space for all of the tested scenarios with $100 \mathrm{fb}^{-1}$, indicating that these points are discoverable (or excludable) with the current LHC Run 2 data set.

Particularly strong sensitivity in distinguishing SM Higgs couplings from contact terms PO points occurs when the contributions to the amplitude from the contact terms interfere destructively with the SM Higgs amplitude [92], as can be seen in figure 1 (left) or figure 2 (left) for $\epsilon_{Z \ell_{L}} \sim-\epsilon_{Z \ell_{R}} \sim-0.23$. In scenarios 5 and 6 , where lepton-flavor universality violation is present, the comparison of the different $H \rightarrow 4 \ell$ channels represents also a very sensitive probe, as long as couplings to electrons and to muons are not too similar. This latter effect is clearly seen comparing the left and right plots in figures 3 and 4 .

It is easy to note that the separation power for different coupling points obeys certain symmetries: the swapping of electrons and muons ( $\mathrm{x}$ with $\mathrm{y}$ ), in figures 3 and 4 , does not change the sensitivity for the scenarios 5 and 6 , while the flip of any coupling sign, in figure 3 , gives an approximately symmetric result. 


\section{Conclusions}

Studies of the Higgs boson in the golden, four-lepton, final state remain important in LHC Run 2 and beyond. A useful toolkit for describing the probed Higgs-to-diboson (and, more generally, $H Z \ell \ell$ ) interactions exists and contains three major methods for parameterizing couplings. These consist in the specification of the most general $H \rightarrow V V$ amplitude compatible with the symmetries assumed (the amplitude approach), the specification of the most general $H \rightarrow V V$ Lagrangian terms consistent with these same symmetries (the Lagrangian approach), and the use of the most general decomposition of the $H \rightarrow 4 \ell$ on-shell amplitude in terms of pseudo-observables (the PO approach). The latter can be considered a generalisation of the two other methods (widely adopted so far in the experimental analyses) able, in particular, to cover also more general BSM frameworks.

In this paper we have compared the use of PO to the other approaches. We showed how to translate between the conventions used by the ATLAS and CMS experiments and in selected theoretical papers, and the parameterization of PO for the four-lepton case specified in ref. [156]. We have also provided projections for the LHC Run 2 sensitivity to departures from SM couplings in this channel, and we have analyzed the role of kinematic distributions and (ratios of) rates in such $H \rightarrow 4 \ell$ studies. In doing so, we have both demonstrated the use of PO and illustrated their relationship to other parametrization methods.

Our work here has aimed at expanding the experimental and phenomenological toolbox for studies of the four-lepton final state. This channel, which provides a treasure trove of information about the underlying coupling of the Higgs to bosons (and leptons), will play an justifiably important role in the LHC physics program for years to come. It is our hope that subsequent studies of the golden channel will lead to a deeper understanding of the Higgs and, perhaps, help inaugurate a golden age of BSM discovery in particle physics.

\section{Acknowledgments}

JL, AK, KM, PM, GM, SM, and AR thank their CMS colleagues for useful discussions. Work supported by DOE Grants No. DE-SC0010296 and DE-SC0010504; the Swiss National Science Foundation under contract 200021-159720; and a Marie Skłodowska-Curie Individual Fellowship of the European Commission's Horizon 2020 Programme under contract number 745954 Tau-SYNERGIES. The research at Cornell was supported by National Science Foundation grant PHY-1607126. MP is supported by the National Research Foundation of Korea (NRF) grant funded by the Korea government (MSIT) (No. NRF2018R1C1B6006572). This document was prepared in part using the resources of the Fermi National Accelerator Laboratory (Fermilab), a U.S. Department of Energy, Office of Science, HEP User Facility. Fermilab is managed by Fermi Research Alliance, LLC (FRA), acting under Contract No. DE-AC02-07CH11359. This work was performed in part at the Aspen Center for Physics, which is supported by National Science Foundation grant PHY-1066293.

Open Access. This article is distributed under the terms of the Creative Commons Attribution License (CC-BY 4.0), which permits any use, distribution and reproduction in any medium, provided the original author(s) and source are credited. 


\section{References}

[1] ATLAS collaboration, Observation of a new particle in the search for the Standard Model Higgs boson with the ATLAS detector at the LHC, Phys. Lett. B 716 (2012) 1 [arXiv: 1207.7214] [INSPIRE].

[2] CMS collaboration, Observation of a new boson at a mass of $125 \mathrm{GeV}$ with the CMS experiment at the LHC, Phys. Lett. B 716 (2012) 30 [arXiv:1207.7235] [INSPIRE].

[3] ATLAS collaboration, Limits on the production of the Standard Model Higgs boson in pp collisions at $\sqrt{s}=7$ TeV with the ATLAS detector, Eur. Phys. J. C 71 (2011) 1728 [arXiv:1106.2748] [INSPIRE].

[4] ATLAS collaboration, Search for the Standard Model Higgs boson in the decay channel $H \rightarrow Z Z^{(*)} \rightarrow 4 \ell$ with the ATLAS detector, Phys. Lett. B 705 (2011) 435 [arXiv:1109.5945] [INSPIRE].

[5] ATLAS collaboration, Combined search for the Standard Model Higgs boson using up to $4.9 \mathrm{fb}^{-1}$ of pp collision data at $\sqrt{\mathrm{s}}=7 \mathrm{TeV}$ with the ATLAS detector at the LHC, Phys. Lett. B 710 (2012) 49 [arXiv:1202.1408] [INSPIRE].

[6] ATLAS collaboration, Search for the Standard Model Higgs boson in the decay channel $H \rightarrow Z Z^{(*)} \rightarrow 4 \ell$ with $4.8 \mathrm{fb}^{-1}$ of $p p$ collision data at $\sqrt{s}=7$ TeV with ATLAS, Phys. Lett. B 710 (2012) 383 [arXiv:1202.1415] [INSPIRE].

[7] CMS collaboration, Combined results of searches for the Standard Model Higgs boson in pp collisions at $\sqrt{s}=7 \mathrm{TeV}$, Phys. Lett. B 710 (2012) 26 [arXiv:1202.1488] [INSPIRE].

[8] CMS collaboration, Search for the Standard Model Higgs boson in the decay channel $H \rightarrow Z Z \rightarrow 4 \ell$ in pp collisions at $\sqrt{s}=7$ TeV, Phys. Rev. Lett. 108 (2012) 111804 [arXiv: 1202.1997] [INSPIRE].

[9] ATLAS collaboration, Combined search for the Standard Model Higgs boson in pp collisions at $\sqrt{s}=7 \mathrm{TeV}$ with the ATLAS detector, Phys. Rev. D 86 (2012) 032003 [arXiv:1207.0319] [INSPIRE].

[10] CMS collaboration, Observation of $Z$ decays to four leptons with the CMS detector at the LHC, JHEP 12 (2012) 034 [arXiv:1210.3844] [INSPIRE].

[11] ATLAS collaboration, A particle consistent with the Higgs boson observed with the ATLAS detector at the Large Hadron Collider, Science 338 (2012) 1576 [INSPIRE].

[12] CMS collaboration, Search for exotic resonances decaying into $W Z / Z Z$ in pp collisions at $\sqrt{s}=7 \mathrm{Te}$, JHEP 02 (2013) 036 [arXiv:1211.5779] [INSPIRE].

[13] CMS collaboration, Study of the mass and spin-parity of the Higgs boson candidate via its decays to Z boson pairs, Phys. Rev. Lett. 110 (2013) 081803 [arXiv:1212.6639] [INSPIRE].

[14] CMS collaboration, Observation of a new boson with mass near 125 GeV in pp collisions at $\sqrt{s}=7$ and $8 \mathrm{TeV}$, JHEP 06 (2013) 081 [arXiv: 1303.4571] [INSPIRE].

[15] CMS collaboration, Search for a Standard-Model-like Higgs boson with a mass in the range 145 to $1000 \mathrm{GeV}$ at the LHC, Eur. Phys. J. C 73 (2013) 2469 [arXiv:1304.0213] [InSPIRE].

[16] ATLAS collaboration, Measurements of Higgs boson production and couplings in diboson final states with the ATLAS detector at the LHC, Phys. Lett. B 726 (2013) 88 [Erratum ibid. B 734 (2014) 406] [arXiv:1307.1427] [INSPIRE]. 
[17] ATLAS collaboration, Evidence for the spin-0 nature of the Higgs boson using ATLAS data, Phys. Lett. B 726 (2013) 120 [arXiv:1307.1432] [INSPIRE].

[18] CMS collaboration, Measurement of the properties of a Higgs boson in the four-lepton final state, Phys. Rev. D 89 (2014) 092007 [arXiv:1312.5353] [InSPIRE].

[19] CMS collaboration, Constraints on the Higgs boson width from off-shell production and decay to Z-boson pairs, Phys. Lett. B 736 (2014) 64 [arXiv:1405.3455] [InSPIRE].

[20] ATLAS collaboration, Measurement of the Higgs boson mass from the $H \rightarrow \gamma \gamma$ and $H \rightarrow Z Z^{*} \rightarrow 4 \ell$ channels with the ATLAS detector using $25 \mathrm{fb}^{-1}$ of pp collision data, Phys. Rev. D 90 (2014) 052004 [arXiv: 1406.3827] [InSPIRE].

[21] ATLAS collaboration, Fiducial and differential cross sections of Higgs boson production measured in the four-lepton decay channel in pp collisions at $\sqrt{s}=8 \mathrm{TeV}$ with the ATLAS detector, Phys. Lett. B 738 (2014) 234 [arXiv:1408.3226] [INSPIRE].

[22] CMS collaboration, Constraints on the spin-parity and anomalous HVV couplings of the Higgs boson in proton collisions at 7 and 8 TeV, Phys. Rev. D 92 (2015) 012004 [arXiv: 1411.3441] [INSPIRE].

[23] CMS collaboration, Precise determination of the mass of the Higgs boson and tests of compatibility of its couplings with the Standard Model predictions using proton collisions at 7 and $8 \mathrm{TeV}$, Eur. Phys. J. C 75 (2015) 212 [arXiv:1412.8662] [INSPIRE].

[24] ATLAS collaboration, Constraints on the off-shell Higgs boson signal strength in the high-mass $Z Z$ and $W W$ final states with the ATLAS detector, Eur. Phys. J. C 75 (2015) 335 [arXiv: 1503.01060] [INSPIRE].

[25] ATLAS and CMS collaborations, Combined measurement of the Higgs boson mass in pp collisions at $\sqrt{s}=7$ and $8 \mathrm{TeV}$ with the ATLAS and CMS experiments, Phys. Rev. Lett. 114 (2015) 191803 [arXiv: 1503.07589] [INSPIRE].

[26] CMS collaboration, Search for a Higgs boson in the mass range from 145 to $1000 \mathrm{GeV}$ decaying to a pair of $W$ or $Z$ bosons, JHEP 10 (2015) 144 [arXiv:1504.00936] [INSPIRE].

[27] ATLAS collaboration, Measurements of the total and differential Higgs boson production cross sections combining the $H \rightarrow \gamma \gamma$ and $H \rightarrow Z Z^{*} \rightarrow 4 \ell$ decay channels at $\sqrt{s}=8 \mathrm{TeV}$ with the ATLAS detector, Phys. Rev. Lett. 115 (2015) 091801 [arXiv:1504.05833] [INSPIRE].

[28] ATLAS collaboration, Search for new light gauge bosons in Higgs boson decays to four-lepton final states in pp collisions at $\sqrt{s}=8 \mathrm{TeV}$ with the ATLAS detector at the LHC, Phys. Rev. D 92 (2015) 092001 [arXiv:1505. 07645] [INSPIRE].

[29] CMS collaboration, A search for pair production of new light bosons decaying into muons, Phys. Lett. B 752 (2016) 146 [arXiv: 1506.00424] [INSPIRE].

[30] ATLAS collaboration, Study of the spin and parity of the Higgs boson in diboson decays with the ATLAS detector, Eur. Phys. J. C 75 (2015) 476 [Erratum ibid. C 76 (2016) 152] [arXiv: 1506. 05669] [INSPIRE].

[31] ATLAS collaboration, Search for an additional, heavy Higgs boson in the $H \rightarrow Z Z$ decay channel at $\sqrt{s}=8 \mathrm{TeV}$ in pp collision data with the ATLAS detector, Eur. Phys. J. C 76 (2016) 45 [arXiv: 1507.05930] [inSPIRE].

[32] CMS collaboration, Limits on the Higgs boson lifetime and width from its decay to four charged leptons, Phys. Rev. D 92 (2015) 072010 [arXiv:1507.06656] [INSPIRE]. 
[33] CMS collaboration, Measurement of differential and integrated fiducial cross sections for Higgs boson production in the four-lepton decay channel in pp collisions at $\sqrt{s}=7$ and $8 \mathrm{TeV}$, JHEP 04 (2016) 005 [arXiv: 1512.08377] [INSPIRE].

[34] CMS collaboration, Combined search for anomalous pseudoscalar $H V V$ couplings in $V H(H \rightarrow b \bar{b})$ production and $H \rightarrow V V$ decay, Phys. Lett. B 759 (2016) 672 [arXiv: 1602.04305] [INSPIRE].

[35] CMS collaboration, Search for Higgs boson off-shell production in proton-proton collisions at 7 and $8 \mathrm{TeV}$ and derivation of constraints on its total decay width, JHEP 09 (2016) 051 [arXiv: 1605. 02329] [INSPIRE].

[36] ATLAS and CMS collaborations, Measurements of the Higgs boson production and decay rates and constraints on its couplings from a combined ATLAS and CMS analysis of the LHC pp collision data at $\sqrt{s}=7$ and 8 TeV, JHEP 08 (2016) 045 [arXiv:1606.02266] [INSPIRE].

[37] ATLAS collaboration, Searches for heavy diboson resonances in pp collisions at $\sqrt{s}=13 \mathrm{TeV}$ with the ATLAS detector, JHEP 09 (2016) 173 [arXiv:1606.04833] [INSPIRE].

[38] CMS collaboration, Measurements of properties of the Higgs boson decaying into the four-lepton final state in pp collisions at $\sqrt{s}=13$ TeV, JHEP 11 (2017) 047 [arXiv: 1706. 09936] [INSPIRE].

[39] CMS collaboration, Constraints on anomalous Higgs boson couplings using production and decay information in the four-lepton final state, Phys. Lett. B 775 (2017) 1 [arXiv: 1707.00541] [INSPIRE].

[40] ATLAS collaboration, Measurement of the Higgs boson coupling properties in the $H \rightarrow Z Z^{*} \rightarrow 4 \ell$ decay channel at $\sqrt{s}=13 \mathrm{TeV}$ with the ATLAS detector, JHEP 03 (2018) 095 [arXiv: 1712. 02304] [INSPIRE].

[41] CMS collaboration, Search for a new scalar resonance decaying to a pair of $Z$ bosons in proton-proton collisions at $\sqrt{s}=13 \mathrm{TeV}$, JHEP 06 (2018) 127 [arXiv:1804.01939] [INSPIRE].

[42] J.R. Dell'Aquila and C.A. Nelson, $P$ or $C P$ determination by sequential decays: $V 1 V 2$ modes with decays into $\bar{\ell}_{A} \ell_{B}$ and/or $\bar{q}_{A} q_{B}$, Phys. Rev. D 33 (1986) 80 [INSPIRE].

[43] C.A. Nelson, Correlation between decay planes in Higgs boson decays into $W$ pair (into $Z$ pair), Phys. Rev. D 37 (1988) 1220 [INSPIRE].

[44] B.A. Kniehl, The Higgs boson decay H $\rightarrow$ Zgg, Phys. Lett. B 244 (1990) 537 [inSPIRE].

[45] A. Soni and R.M. Xu, Probing CP-violation via Higgs decays to four leptons, Phys. Rev. D 48 (1993) 5259 [hep-ph/9301225] [INSPIRE].

[46] D. Chang, W.-Y. Keung and I. Phillips, CP odd correlation in the decay of neutral Higgs boson into $Z Z, W^{+} W^{-}$, or t $\bar{t}$, Phys. Rev. D 48 (1993) 3225 [hep-ph/9303226] [INSPIRE].

[47] V.D. Barger, K.-M. Cheung, A. Djouadi, B.A. Kniehl and P.M. Zerwas, Higgs bosons: intermediate mass range at $e^{+} e^{-}$colliders, Phys. Rev. D 49 (1994) 79 [hep-ph/9306270] [INSPIRE].

[48] T. Arens and L.M. Sehgal, Energy spectra and energy correlations in the decay $H \rightarrow Z Z \rightarrow \mu^{+} \mu^{-} \mu^{+} \mu^{-}, Z$. Phys. C 66 (1995) 89 [hep-ph/9409396] [INSPIRE]. 
[49] S.Y. Choi, D.J. Miller, M.M. Muhlleitner and P.M. Zerwas, Identifying the Higgs spin and parity in decays to $Z$ pairs, Phys. Lett. B 553 (2003) 61 [hep-ph/0210077] [INSPIRE].

[50] B.C. Allanach, K. Odagiri, M.J. Palmer, M.A. Parker, A. Sabetfakhri and B.R. Webber, Exploring small extra dimensions at the Large Hadron Collider, JHEP 12 (2002) 039 [hep-ph/0211205] [INSPIRE].

[51] C.P. Buszello, I. Fleck, P. Marquard and J.J. van der Bij, Prospective analysis of spin- and $C P$-sensitive variables in $H \rightarrow Z Z \rightarrow \ell_{1}^{+} \ell_{1}^{-} \ell_{2}^{+} \ell_{2}^{-}$at the LHC, Eur. Phys. J. C 32 (2004) 209 [hep-ph/0212396] [INSPIRE].

[52] S. Schalla, Study on the measurement of the CP-eigenstate of Higgs bosons with the CMS experiment at the LHC, Diploma, IEKP-KA-2004-14, Karlsruhe U., Karlsruhe, Germany, (2004) [INSPIRE].

[53] R.M. Godbole, D.J. Miller and M.M. Muhlleitner, Aspects of CP-violation in the $H Z Z$ coupling at the LHC, JHEP 12 (2007) 031 [arXiv: 0708. 0458] [INSPIRE].

[54] V.A. Kovalchuk, Model-independent analysis of CP-violation effects in decays of the Higgs boson into a pair of the $W$ and $Z$ bosons, J. Exp. Theor. Phys. 107 (2008) 774 [INSPIRE].

[55] W.-Y. Keung, I. Low and J. Shu, Landau-Yang theorem and decays of a $Z^{\prime}$ boson into two $Z$ bosons, Phys. Rev. Lett. 101 (2008) 091802 [arXiv:0806.2864] [InSPIRE].

[56] O. Antipin and A. Soni, Towards establishing the spin of warped gravitons, JHEP 10 (2008) 018 [arXiv: 0806.3427] [INSPIRE].

[57] Q.-H. Cao, C.B. Jackson, W.-Y. Keung, I. Low and J. Shu, The Higgs mechanism and loop-induced decays of a scalar into two Z bosons, Phys. Rev. D 81 (2010) 015010 [arXiv: 0911.3398] [INSPIRE].

[58] Y. Gao, A.V. Gritsan, Z. Guo, K. Melnikov, M. Schulze and N.V. Tran, Spin determination of single-produced resonances at hadron colliders, Phys. Rev. D 81 (2010) 075022 [arXiv:1001.3396] [INSPIRE].

[59] A. De Rujula, J. Lykken, M. Pierini, C. Rogan and M. Spiropulu, Higgs look-alikes at the LHC, Phys. Rev. D 82 (2010) 013003 [arXiv: 1001.5300] [inSPIRE].

[60] C. Englert, C. Hackstein and M. Spannowsky, Measuring spin and CP from semi-hadronic ZZ decays using jet substructure, Phys. Rev. D 82 (2010) 114024 [arXiv:1010.0676] [INSPIRE].

[61] A. Matsuzaki and H. Tanaka, Determination of the Higgs CP property in hadron colliders, arXiv: 1101.2104 [INSPIRE].

[62] U. De Sanctis, M. Fabbrichesi and A. Tonero, Telling the spin of the 'Higgs boson' at the LHC, Phys. Rev. D 84 (2011) 015013 [arXiv:1103.1973] [InSPIRE].

[63] H.E. Logan and J.Z. Salvail, Model-independent Higgs coupling measurements at the LHC using the $H \rightarrow Z Z \rightarrow 4 \ell$ lineshape, Phys. Rev. D 84 (2011) 073001 [arXiv:1107.4342] [INSPIRE].

[64] J.S. Gainer, K. Kumar, I. Low and R. Vega-Morales, Improving the sensitivity of Higgs boson searches in the golden channel, JHEP 11 (2011) 027 [arXiv:1108.2274] [INSPIRE].

[65] I. Low, P. Schwaller, G. Shaughnessy and C.E.M. Wagner, The dark side of the Higgs boson, Phys. Rev. D 85 (2012) 015009 [arXiv:1110.4405] [InSPIRE].

[66] C. Englert, M. Spannowsky and M. Takeuchi, Measuring Higgs CP and couplings with hadronic event shapes, JHEP 06 (2012) 108 [arXiv:1203.5788] [INSPIRE]. 
[67] J.M. Campbell, W.T. Giele and C. Williams, The matrix element method at next-to-leading order, JHEP 11 (2012) 043 [arXiv: 1204.4424] [INSPIRE].

[68] J.M. Campbell, W.T. Giele and C. Williams, Extending the matrix element method to next-to-leading order, in Proceedings, $47^{\text {th }}$ Rencontres de Moriond on QCD and high energy interactions, FERMILAB-CONF-12-176-T, La Thuile, Italy, 10-17 March 2012, pg. 319 [arXiv: 1205.3434] [INSPIRE].

[69] N. Kauer and G. Passarino, Inadequacy of zero-width approximation for a light Higgs boson signal, JHEP 08 (2012) 116 [arXiv:1206.4803] [INSPIRE].

[70] B.A. Kniehl and O.L. Veretin, Low-mass Higgs decays to four leptons at one loop and beyond, Phys. Rev. D 86 (2012) 053007 [arXiv:1206.7110] [INSPIRE].

[71] J.W. Moffat, Identification of the $125 \mathrm{GeV}$ resonance as a pseudoscalar quarkonium meson, arXiv: 1207.6015 [INSPIRE].

[72] B. Coleppa, K. Kumar and H.E. Logan, Can the $126 \mathrm{GeV}$ boson be a pseudoscalar?, Phys. Rev. D 86 (2012) 075022 [arXiv: 1208.2692] [INSPIRE].

[73] S. Bolognesi et al., On the spin and parity of a single-produced resonance at the LHC, Phys. Rev. D 86 (2012) 095031 [arXiv: 1208.4018] [InSPIRE].

[74] R. Boughezal, T.J. LeCompte and F. Petriello, Single-variable asymmetries for measuring the 'Higgs' boson spin and CP properties, arXiv:1208.4311 [INSPIRE].

[75] D. Stolarski and R. Vega-Morales, Directly measuring the tensor structure of the scalar coupling to gauge bosons, Phys. Rev. D 86 (2012) 117504 [arXiv:1208.4840] [INSPIRE].

[76] P. Cea, Comment on the evidence of the Higgs boson at LHC, arXiv:1209.3106 [INSPIRE].

[77] J. Kumar, A. Rajaraman and D. Yaylali, Spin determination for fermiophobic bosons, Phys. Rev. D 86 (2012) 115019 [arXiv: 1209.5432] [InSPIRE].

[78] C.-Q. Geng, D. Huang, Y. Tang and Y.-L. Wu, Note on 125 GeV spin-2 particle, Phys. Lett. B 719 (2013) 164 [arXiv:1210.5103] [INSPIRE].

[79] P. Avery et al., Precision studies of the Higgs boson decay channel $H \rightarrow Z Z \rightarrow 4 \ell$ with MEKD, Phys. Rev. D 87 (2013) 055006 [arXiv:1210.0896] [INSPIRE].

[80] E. Massó and V. Sanz, Limits on anomalous couplings of the Higgs boson to electroweak gauge bosons from LEP and the LHC, Phys. Rev. D 87 (2013) 033001 [arXiv:1211.1320] [INSPIRE].

[81] Y. Chen, N. Tran and R. Vega-Morales, Scrutinizing the Higgs signal and background in the $2 e 2 \mu$ golden channel, JHEP 01 (2013) 182 [arXiv:1211.1959] [INSPIRE].

[82] A. Menon, T. Modak, D. Sahoo, R. Sinha and H.-Y. Cheng, Inferring the nature of the boson at 125-126 GeV, Phys. Rev. D 89 (2014) 095021 [arXiv:1301.5404] [InSPIRE].

[83] S. Kanemura, M. Kikuchi and K. Yagyu, Probing exotic Higgs sectors from the precise measurement of Higgs boson couplings, Phys. Rev. D 88 (2013) 015020 [arXiv:1301.7303] [INSPIRE].

[84] J.S. Gainer, J. Lykken, K.T. Matchev, S. Mrenna and M. Park, Geolocating the Higgs boson candidate at the LHC, Phys. Rev. Lett. 111 (2013) 041801 [arXiv:1304.4936] [INSPIRE].

[85] G. Isidori, A.V. Manohar and M. Trott, Probing the nature of the Higgs-like boson via $h \rightarrow V \mathcal{F}$ decays, Phys. Lett. B 728 (2014) 131 [arXiv:1305.0663] [INSPIRE]. 
[86] J. Frank, M. Rauch and D. Zeppenfeld, Higgs spin determination in the $W W$ channel and beyond, Eur. Phys. J. C 74 (2014) 2918 [arXiv:1305.1883] [InSPIRE].

[87] B. Grinstein, C.W. Murphy and D. Pirtskhalava, Searching for new physics in the three-body decays of the Higgs-like particle, JHEP 10 (2013) 077 [arXiv:1305.6938] [INSPIRE].

[88] F. Caola and K. Melnikov, Constraining the Higgs boson width with $Z Z$ production at the LHC, Phys. Rev. D 88 (2013) 054024 [arXiv:1307.4935] [InSPIRE].

[89] S. Banerjee, S. Mukhopadhyay and B. Mukhopadhyaya, Higher dimensional operators and the LHC Higgs data: the role of modified kinematics, Phys. Rev. D 89 (2014) 053010 [arXiv:1308.4860] [INSPIRE].

[90] Y. Sun, X.-F. Wang and D.-N. Gao, CP mixed property of the Higgs-like particle in the decay channel $h \rightarrow Z Z^{*} \rightarrow 4 \ell$, Int. J. Mod. Phys. A 29 (2014) 1450086 [arXiv:1309.4171] [INSPIRE].

[91] I. Anderson et al., Constraining anomalous $H V V$ interactions at proton and lepton colliders, Phys. Rev. D 89 (2014) 035007 [arXiv:1309.4819] [InSPIRE].

[92] M. Chen et al., The role of interference in unraveling the $Z Z$-couplings of the newly discovered boson at the LHC, Phys. Rev. D 89 (2014) 034002 [arXiv:1310.1397] [INSPIRE].

[93] G. Buchalla, O. Catà and G. D'Ambrosio, Nonstandard Higgs couplings from angular distributions in $h \rightarrow Z \ell^{+} \ell^{-}$, Eur. Phys. J. C 74 (2014) 2798 [arXiv:1310.2574] [inSPIRE].

[94] Y. Chen and R. Vega-Morales, Extracting effective Higgs couplings in the golden channel, JHEP 04 (2014) 057 [arXiv: 1310.2893] [INSPIRE].

[95] J.M. Campbell, R.K. Ellis and C. Williams, Bounding the Higgs width at the LHC using full analytic results for $g g \rightarrow e^{-} e^{+} \mu^{-} \mu^{+}$, JHEP 04 (2014) 060 [arXiv:1311.3589] [INSPIRE].

[96] D. Curtin et al., Exotic decays of the 125 GeV Higgs boson, Phys. Rev. D 90 (2014) 075004 [arXiv: 1312.4992] [INSPIRE].

[97] Y. Chen, E. Di Marco, J. Lykken, M. Spiropulu, R. Vega-Morales and S. Xie, $8 D$ likelihood effective Higgs couplings extraction framework in $h \rightarrow 4 \ell$, JHEP 01 (2015) 125 [arXiv: 1401.2077] [INSPIRE].

[98] M. González-Alonso and G. Isidori, The $h \rightarrow 4 \ell$ spectrum at low $m_{34}$ : Standard Model vs. light new physics, Phys. Lett. B 733 (2014) 359 [arXiv: 1403.2648] [INSPIRE].

[99] J.S. Gainer, J. Lykken, K.T. Matchev, S. Mrenna and M. Park, Beyond geolocating: constraining higher dimensional operators in $H \rightarrow 4 \ell$ with off-shell production and more, Phys. Rev. D 91 (2015) 035011 [arXiv:1403.4951] [INSPIRE].

[100] Y. Chen, R. Harnik and R. Vega-Morales, Probing the Higgs couplings to photons in $h \rightarrow 4 \ell$ at the LHC, Phys. Rev. Lett. 113 (2014) 191801 [arXiv:1404.1336] [INSPIRE].

[101] A. Falkowski and R. Vega-Morales, Exotic Higgs decays in the golden channel, JHEP 12 (2014) 037 [arXiv: 1405.1095] [INSPIRE].

[102] M. Beneke, D. Boito and Y.-M. Wang, Anomalous Higgs couplings in angular asymmetries of $H \rightarrow Z \ell^{+} \ell^{-}$and $e^{+} e^{-} \rightarrow H Z$, JHEP 11 (2014) 028 [arXiv:1406.1361] [INSPIRE].

[103] D.-N. Gao, A note on Higgs decays into $Z$ boson and $J / \Psi(\Upsilon)$, Phys. Lett. B 737 (2014) 366 [arXiv: 1406.7102] [INSPIRE].

[104] T. Modak, D. Sahoo, R. Sinha, H.-Y. Cheng and T.-C. Yuan, Disentangling the spin-parity of a resonance via the gold-plated decay mode, Chin. Phys. C 40 (2016) 033002 [arXiv:1408.5665] [INSPIRE]. 
[105] Y. Chen, E. Di Marco, J. Lykken, M. Spiropulu, R. Vega-Morales and S. Xie, Technical note for $8 D$ likelihood effective Higgs couplings extraction framework in the golden channel, arXiv: 1410.4817 [INSPIRE].

[106] T. Modak and R. Srivastava, Probing anomalous Higgs couplings in $H \rightarrow Z V$ decays, Mod. Phys. Lett. A 32 (2017) 1750004 [arXiv:1411.2210] [INSPIRE].

[107] M. Beneke, D. Boito and Y.-M. Wang, Signatures of anomalous Higgs couplings in angular asymmetries of $H \rightarrow Z \ell^{+} \ell^{-}$and $e^{+} e^{-} \rightarrow H Z$, Nucl. Part. Phys. Proc. 273-275 (2016) 846 [arXiv: 1411.3942] [INSPIRE].

[108] D. Curtin, R. Essig, S. Gori and J. Shelton, Illuminating dark photons with high-energy colliders, JHEP 02 (2015) 157 [arXiv: 1412.0018] [INSPIRE].

[109] N. Belyaev, R. Konoplich, L.E. Pedersen and K. Prokofiev, Angular asymmetries as a probe for anomalous contributions to HZZ vertex at the LHC, Phys. Rev. D 91 (2015) 115014 [arXiv: 1502.03045] [INSPIRE].

[110] C. Englert, I. Low and M. Spannowsky, On-shell interference effects in Higgs boson final states, Phys. Rev. D 91 (2015) 074029 [arXiv: 1502.04678] [INSPIRE].

[111] Y. Chen, R. Harnik and R. Vega-Morales, New opportunities in $h \rightarrow 4 \ell$, JHEP 09 (2015) 185 [arXiv: 1503.05855] [INSPIRE].

[112] B. Bhattacherjee, T. Modak, S.K. Patra and R. Sinha, Probing Higgs couplings at LHC and beyond, arXiv:1503.08924 [INSPIRE].

[113] S. Furui, Cartan's supersymmetry and the decay of a $H^{0}\left(0^{+}\right)$, arXiv: 1504.03795 [INSPIRE].

[114] M. González-Alonso, A. Greljo, G. Isidori and D. Marzocca, Electroweak bounds on Higgs pseudo-observables and $h \rightarrow 4 \ell$ decays, Eur. Phys. J. C 75 (2015) 341 [arXiv: 1504.04018] [INSPIRE].

[115] T.V. Zagoskin and A. Yu. Korchin, Decays of a neutral particle with zero spin and arbitrary CP parity into two off-mass-shell Z bosons, J. Exp. Theor. Phys. 122 (2016) 663 [arXiv: 1504.07187] [INSPIRE].

[116] S. Banerjee, T. Mandal, B. Mellado and B. Mukhopadhyaya, Cornering dimension-6 HVV interactions at high luminosity LHC: the role of event ratios, JHEP 09 (2015) 057 [arXiv: 1505.00226] [INSPIRE].

[117] Y. Zhou, Constraining the Higgs boson coupling to light quarks in the $H \rightarrow Z Z$ final states, Phys. Rev. D 93 (2016) 013019 [arXiv: 1505.06369] [INSPIRE].

[118] Y. Chen, D. Stolarski and R. Vega-Morales, Golden probe of the top Yukuwa coupling, Phys. Rev. D 92 (2015) 053003 [arXiv: 1505.01168] [INSPIRE].

[119] D. Curtin and C.B. Verhaaren, Discovering uncolored naturalness in exotic Higgs decays, JHEP 12 (2015) 072 [arXiv: 1506.06141] [INSPIRE].

[120] H. Davoudiasl, H.-S. Lee and W.J. Marciano, Low $Q^{2}$ weak mixing angle measurements and rare Higgs decays, Phys. Rev. D 92 (2015) 055005 [arXiv: 1507.00352] [InSPIRE].

[121] A. Djouadi, J. Quevillon and R. Vega-Morales, Into the multi-TeV scale with a Higgs golden ratio, Phys. Lett. B 757 (2016) 412 [arXiv: 1509.03913] [INSPIRE].

[122] A. Delgado, M. Garcia-Pepin, M. Quirós, J. Santiago and R. Vega-Morales, Diphoton and diboson probes of fermiophobic Higgs bosons at the LHC, JHEP 06 (2016) 042 [arXiv: 1603.00962] [INSPIRE]. 
[123] S. Dwivedi, D.K. Ghosh, B. Mukhopadhyaya and A. Shivaji, Distinguishing CP-odd couplings of the Higgs boson to weak boson pairs, Phys. Rev. D 93 (2016) 115039 [arXiv: 1603.06195] [INSPIRE].

[124] F. Caola, M. Dowling, K. Melnikov, R. Röntsch and L. Tancredi, QCD corrections to vector boson pair production in gluon fusion including interference effects with off-shell Higgs at the LHC, JHEP 07 (2016) 087 [arXiv: 1605.04610] [INSPIRE].

[125] Y. Chen, J. Lykken, M. Spiropulu, D. Stolarski and R. Vega-Morales, Golden probe of electroweak symmetry breaking, Phys. Rev. Lett. 117 (2016) 241801 [arXiv: 1608. 02159] [INSPIRE].

[126] I.F. Ginzburg, What tell us LHC data about Higgs boson parity, arXiv:1610.08196 [INSPIRE].

[127] C. Grojean, Physics of the Brout-Englert-Higgs boson: theory, Nucl. Part. Phys. Proc. 273-275 (2016) 11 [INSPIRE].

[128] LHC Higgs Cross Section Working Group collaboration, D. de Florian et al., Handbook of LHC Higgs cross sections: 4. Deciphering the nature of the Higgs sector, arXiv: 1610.07922 [INSPIRE].

[129] T.V. Zagoskin and A. Yu. Korchin, The Higgs boson decay into ZZ decaying to identical fermion pairs, Int. J. Mod. Phys. A 32 (2017) 1750166 [arXiv:1701.06335] [InSPIRE].

[130] J. Chang, K. Cheung, J.S. Lee, C.-T. Lu and J. Park, Measuring properties of a heavy Higgs boson in the $H \rightarrow Z Z \rightarrow 4 \ell$ decay, JHEP 12 (2017) 053 [arXiv: 1708.05583] [INSPIRE].

[131] R. Vega, R. Vega-Morales and K. Xie, The supersymmetric Georgi-Machacek model, JHEP 03 (2018) 168 [arXiv:1711.05329] [INSPIRE].

[132] J. Brehmer, F. Kling, T. Plehn and T.M.P. Tait, Better Higgs-CP tests through information geometry, Phys. Rev. D 97 (2018) 095017 [arXiv:1712.02350] [INSPIRE].

[133] T. Zagoskin and A. Korchin, The Higgs boson ZZ couplings in the Higgs-strahlung at the $I L C$, arXiv: 1804.10011 [INSPIRE].

[134] J. Brehmer, K. Cranmer, G. Louppe and J. Pavez, Constraining effective field theories with machine learning, Phys. Rev. Lett. 121 (2018) 111801 [arXiv:1805.00013] [INSPIRE].

[135] J. Brehmer, K. Cranmer, G. Louppe and J. Pavez, A guide to constraining effective field theories with machine learning, Phys. Rev. D 98 (2018) 052004 [arXiv:1805.00020] [INSPIRE].

[136] M.B. Wise, H. Georgi and S.L. Glashow, SU(5) and the invisible axion, Phys. Rev. Lett. 47 (1981) 402 [INSPIRE].

[137] R.E. Shrock and M. Suzuki, Invisible decays of Higgs bosons, Phys. Lett. B 110 (1982) 250 [INSPIRE].

[138] K. Griest and H.E. Haber, Invisible decays of Higgs bosons in supersymmetric models, Phys. Rev. D 37 (1988) 719 [INSPIRE].

[139] J.C. Romao, F. de Campos and J.W.F. Valle, New Higgs signatures in supersymmetry with spontaneous broken R parity, Phys. Lett. B 292 (1992) 329 [hep-ph/9207269] [INSPIRE].

[140] J.F. Gunion, Detecting an invisibly decaying Higgs boson at a hadron supercollider, Phys. Rev. Lett. 72 (1994) 199 [hep-ph/9309216] [INSPIRE]. 
[141] D. Choudhury and D.P. Roy, Signatures of an invisibly decaying Higgs particle at LHC, Phys. Lett. B 322 (1994) 368 [hep-ph/9312347] [INSPIRE].

[142] S.G. Frederiksen, N. Johnson, G.L. Kane and J. Reid, Detecting invisible Higgs bosons at the CERN Large Hadron Collider, Phys. Rev. D 50 (1994) R4244 [INSPIRE].

[143] A. Djouadi, P. Janot, J. Kalinowski and P.M. Zerwas, SUSY decays of Higgs particles, Phys. Lett. B 376 (1996) 220 [hep-ph/9603368] [INSPIRE].

[144] S.P. Martin and J.D. Wells, Motivation and detectability of an invisibly decaying Higgs boson at the Fermilab Tevatron, Phys. Rev. D 60 (1999) 035006 [hep-ph/9903259] [INSPIRE].

[145] N.D. Christensen and C. Duhr, FeynRules - Feynman rules made easy, Comput. Phys. Commun. 180 (2009) 1614 [arXiv:0806.4194] [InSPIRE].

[146] A. Alloul, N.D. Christensen, C. Degrande, C. Duhr and B. Fuks, FeynRules $2.0-a$ complete toolbox for tree-level phenomenology, Comput. Phys. Commun. 185 (2014) 2250 [arXiv: 1310.1921] [INSPIRE].

[147] A. Pukhov, CalcHEP 2.3: MSSM, structure functions, event generation, batchs and generation of matrix elements for other packages, hep-ph/0412191 [INSPIRE].

[148] A. Belyaev, N.D. Christensen and A. Pukhov, CalcHEP 3.4 for collider physics within and beyond the Standard Model, Comput. Phys. Commun. 184 (2013) 1729 [arXiv:1207.6082] [INSPIRE].

[149] E.E. Boos, M.N. Dubinin, V.A. Ilyin, A.E. Pukhov and V.I. Savrin, CompHEP: specialized package for automatic calculations of elementary particle decays and collisions, hep-ph/9503280 [INSPIRE].

[150] A. Pukhov et al., CompHEP: a package for evaluation of Feynman diagrams and integration over multiparticle phase space, hep-ph/9908288 [INSPIRE].

[151] ComPHEP collaboration, E. Boos et al., CompHEP 4.4: automatic computations from Lagrangians to events, Nucl. Instrum. Meth. A 534 (2004) 250 [hep-ph/0403113] [INSPIRE].

[152] T. Stelzer and W.F. Long, Automatic generation of tree level helicity amplitudes, Comput. Phys. Commun. 81 (1994) 357 [hep-ph/9401258] [INSPIRE].

[153] F. Maltoni and T. Stelzer, MadEvent: automatic event generation with MadGraph, JHEP 02 (2003) 027 [hep-ph/0208156] [INSPIRE].

[154] J. Alwall et al., MadGraph/MadEvent v4: the new web generation, JHEP 09 (2007) 028 [arXiv:0706.2334] [INSPIRE].

[155] J. Alwall, M. Herquet, F. Maltoni, O. Mattelaer and T. Stelzer, MadGraph 5: going beyond, JHEP 06 (2011) 128 [arXiv:1106.0522] [InSPIRE].

[156] M. González-Alonso, A. Greljo, G. Isidori and D. Marzocca, Pseudo-observables in Higgs decays, Eur. Phys. J. C 75 (2015) 128 [arXiv:1412.6038] [InSPIRE].

[157] A. David and G. Passarino, Through precision straits to next Standard Model heights, Rev. Phys. 1 (2016) 13 [arXiv:1510.00414] [INSPIRE].

[158] D. Yu. Bardin, M. Grunewald and G. Passarino, Precision calculation project report, hep-ph/9902452 [INSPIRE].

[159] SLD Electroweak Group, DelPhi, ALEPH, SLD, SLD Heavy Flavour Group, OPAL, LEP Electroweak Working Group and L3 collaborations, S. Schael et al., Precision electroweak measurements on the $Z$ resonance, Phys. Rept. 427 (2006) 257 [hep-ex/0509008] [INSPIRE]. 
[160] M. Bordone, A. Greljo, G. Isidori, D. Marzocca and A. Pattori, Higgs pseudo observables and radiative corrections, Eur. Phys. J. C 75 (2015) 385 [arXiv:1507.02555] [INSPIRE].

[161] R. Gomez-Ambrosio, Effective field theories and pseudo-observables in the quest for physics beyond the Standard Model, PoS (PLANCK 2015) 049 [arXiv: 1510.02233] [INSPIRE].

[162] A. Greljo, G. Isidori, J.M. Lindert and D. Marzocca, Pseudo-observables in electroweak Higgs production, Eur. Phys. J. C 76 (2016) 158 [arXiv:1512.06135] [INSPIRE].

[163] A. Greljo, G. Isidori, J.M. Lindert, D. Marzocca and H. Zhang, Electroweak Higgs production with HiggsPO at NLO QCD, Eur. Phys. J. C 77 (2017) 838 [arXiv: 1710.04143] [INSPIRE].

[164] N. Kauer, Inadequacy of zero-width approximation for a light Higgs boson signal, Mod. Phys. Lett. A 28 (2013) 1330015 [arXiv:1305.2092] [INSPIRE].

[165] J.M. Campbell, R.K. Ellis and C. Williams, Bounding the Higgs width at the LHC: complementary results from $H \rightarrow W W$, Phys. Rev. D 89 (2014) 053011 [arXiv:1312.1628] [INSPIRE].

[166] G. Passarino, Higgs CAT, Eur. Phys. J. C 74 (2014) 2866 [arXiv:1312.2397] [InSPIRE].

[167] C. Englert and M. Spannowsky, Limitations and opportunities of off-shell coupling measurements, Phys. Rev. D 90 (2014) 053003 [arXiv: 1405.0285] [INSPIRE].

[168] I. Brivio, O.J.P. Éboli, M.B. Gavela, M.C. Gonzalez-Garcia, L. Merlo and S. Rigolin, Higgs ultraviolet softening, JHEP 12 (2014) 004 [arXiv:1405.5412] [INSPIRE].

[169] G. Cacciapaglia, A. Deandrea, G. Drieu La Rochelle and J.-B. Flament, Higgs couplings: disentangling new physics with off-shell measurements, Phys. Rev. Lett. 113 (2014) 201802 [arXiv:1406.1757] [INSPIRE].

[170] A. Azatov, C. Grojean, A. Paul and E. Salvioni, Taming the off-shell Higgs boson, Zh. Eksp. Teor. Fiz. 147 (2015) 410 [J. Exp. Theor. Phys. 120 (2015) 354] [arXiv:1406.6338] [INSPIRE].

[171] J.M. Campbell, R.K. Ellis, E. Furlan and R. Röntsch, Interference effects for Higgs boson mediated Z-pair plus jet production, Phys. Rev. D 90 (2014) 093008 [arXiv:1409.1897] [INSPIRE].

[172] C. Englert, Y. Soreq and M. Spannowsky, Off-shell Higgs coupling measurements in BSM scenarios, JHEP 05 (2015) 145 [arXiv:1410.5440] [INSPIRE].

[173] M. Buschmann, D. Goncalves, S. Kuttimalai, M. Schonherr, F. Krauss and T. Plehn, Mass effects in the Higgs-gluon coupling: boosted vs off-shell production, JHEP 02 (2015) 038 [arXiv:1410.5806] [INSPIRE].

[174] H.E. Logan, Hiding a Higgs width enhancement from off-shell $g g\left(\rightarrow h^{*}\right) \rightarrow Z Z$ measurements, Phys. Rev. D 92 (2015) 075038 [arXiv:1412.7577] [INSPIRE].

[175] N. Kauer, Off-shell Higgs signal and total width determination at the LHC, PoS (FFP14)114, (2016) [arXiv: 1502 .02581] [INSPIRE].

[176] S. Liebler, G. Moortgat-Pick and G. Weiglein, Off-shell effects in Higgs processes at a linear collider and implications for the LHC, JHEP 06 (2015) 093 [arXiv: 1502.07970] [INSPIRE].

[177] C.S. Li, H.T. Li, D.Y. Shao and J. Wang, Soft gluon resummation in the signal-background interference process of $g g\left(\rightarrow h^{*}\right) \rightarrow Z Z$, JHEP 08 (2015) 065 [arXiv:1504.02388] [INSPIRE]. 
[178] C. Englert, M. McCullough and M. Spannowsky, Combining LEP and LHC to bound the Higgs width, Nucl. Phys. B 902 (2016) 440 [arXiv: 1504.02458] [INSPIRE].

[179] F. Campanario, M. Rauch and S. Sapeta, $Z Z$ production at high transverse momenta beyond NLO QCD, JHEP 08 (2015) 070 [arXiv: 1504.05588] [INSPIRE].

[180] A. Azatov, R. Contino and J. Galloway, Model-independent bounds on a light Higgs, JHEP 04 (2012) 127 [Erratum ibid. 04 (2013) 140] [arXiv:1202.3415] [INSPIRE].

[181] M. Klute, R. Lafaye, T. Plehn, M. Rauch and D. Zerwas, Measuring Higgs couplings from LHC data, Phys. Rev. Lett. 109 (2012) 101801 [arXiv:1205.2699] [INSPIRE].

[182] M. Montull and F. Riva, Higgs discovery: the beginning or the end of natural EWSB?, JHEP 11 (2012) 018 [arXiv:1207.1716] [INSPIRE].

[183] J.R. Espinosa, C. Grojean, M. Muhlleitner and M. Trott, First glimpses at Higgs' face, JHEP 12 (2012) 045 [arXiv: 1207.1717 ] [INSPIRE].

[184] D. Carmi, A. Falkowski, E. Kuflik, T. Volansky and J. Zupan, Higgs after the discovery: a status report, JHEP 10 (2012) 196 [arXiv:1207.1718] [INSPIRE].

[185] A. Freitas and P. Schwaller, Higgs CP properties from early LHC data, Phys. Rev. D 87 (2013) 055014 [arXiv:1211.1980] [inSPIRE].

[186] P.P. Giardino, K. Kannike, I. Masina, M. Raidal and A. Strumia, The universal Higgs fit, JHEP 05 (2014) 046 [arXiv: 1303.3570] [INSPIRE].

[187] J. Ellis and T. You, Updated global analysis of Higgs couplings, JHEP 06 (2013) 103 [arXiv: 1303.3879] [INSPIRE].

[188] W. Buchmüller and D. Wyler, Effective Lagrangian analysis of new interactions and flavor conservation, Nucl. Phys. B 268 (1986) 621 [INSPIRE].

[189] K. Hagiwara, R.D. Peccei, D. Zeppenfeld and K. Hikasa, Probing the weak boson sector in $e^{+} e^{-} \rightarrow W^{+} W^{-}$, Nucl. Phys. B 282 (1987) 253 [INSPIRE].

[190] G.F. Giudice, C. Grojean, A. Pomarol and R. Rattazzi, The strongly-interacting light Higgs, JHEP 06 (2007) 045 [hep-ph/0703164] [INSPIRE].

[191] B. Grzadkowski, M. Iskrzynski, M. Misiak and J. Rosiek, Dimension-six terms in the Standard Model Lagrangian, JHEP 10 (2010) 085 [arXiv: 1008.4884] [INSPIRE].

[192] F. Bonnet, M.B. Gavela, T. Ota and W. Winter, Anomalous Higgs couplings at the LHC and their theoretical interpretation, Phys. Rev. D 85 (2012) 035016 [arXiv:1105.5140] [INSPIRE].

[193] T. Corbett, O.J.P. Éboli, J. Gonzalez-Fraile and M.C. Gonzalez-Garcia, Constraining anomalous Higgs interactions, Phys. Rev. D 86 (2012) 075013 [arXiv:1207.1344] [INSPIRE].

[194] J. Ellis and T. You, Global analysis of the Higgs candidate with mass $125 \mathrm{GeV}$, JHEP 09 (2012) 123 [arXiv:1207.1693] [INSPIRE].

[195] G. Cacciapaglia, A. Deandrea, G. Drieu La Rochelle and J.-B. Flament, Higgs couplings beyond the Standard Model, JHEP 03 (2013) 029 [arXiv:1210.8120] [InSPIRE].

[196] G. Bélanger, B. Dumont, U. Ellwanger, J.F. Gunion and S. Kraml, Higgs couplings at the end of 2012, JHEP 02 (2013) 053 [arXiv:1212.5244] [INSPIRE].

[197] T. Corbett, O.J.P. Eboli, J. Gonzalez-Fraile and M.C. Gonzalez-Garcia, Robust determination of the Higgs couplings: power to the data, Phys. Rev. D 87 (2013) 015022 [arXiv:1211.4580] [INSPIRE]. 
[198] C. Grojean, E.E. Jenkins, A.V. Manohar and M. Trott, Renormalization group scaling of Higgs operators and $\Gamma(h \rightarrow \gamma \gamma)$, JHEP 04 (2013) 016 [arXiv: 1301.2588] [INSPIRE].

[199] K. Cheung, J.S. Lee and P.-Y. Tseng, Higgs precision (Higgcision) era begins, JHEP 05 (2013) 134 [arXiv: 1302.3794] [INSPIRE].

[200] J. Elias-Miró, J.R. Espinosa, E. Masso and A. Pomarol, Renormalization of dimension-six operators relevant for the Higgs decays $h \rightarrow \gamma \gamma, \gamma Z$, JHEP 08 (2013) 033 [arXiv: 1302.5661] [INSPIRE].

[201] A. Falkowski, F. Riva and A. Urbano, Higgs at last, JHEP 11 (2013) 111 [arXiv: 1303.1812] [INSPIRE].

[202] R. Contino, M. Ghezzi, C. Grojean, M. Muhlleitner and M. Spira, Effective Lagrangian for a light Higgs-like scalar, JHEP 07 (2013) 035 [arXiv:1303.3876] [INSPIRE].

[203] A. Djouadi and G. Moreau, The couplings of the Higgs boson and its CP properties from fits of the signal strengths and their ratios at the $7+8 T e V$ LHC, Eur. Phys. J. C 73 (2013) 2512 [arXiv: 1303.6591] [INSPIRE].

[204] T. Corbett, O.J.P. Éboli, J. Gonzalez-Fraile and M.C. Gonzalez-Garcia, Determining triple gauge boson couplings from Higgs data, Phys. Rev. Lett. 111 (2013) 011801 [arXiv:1304.1151] [INSPIRE].

[205] B. Dumont, S. Fichet and G. von Gersdorff, A Bayesian view of the Higgs sector with higher dimensional operators, JHEP 07 (2013) 065 [arXiv: 1304.3369] [INSPIRE].

[206] P. Bechtle, S. Heinemeyer, O. Stål, T. Stefaniak and G. Weiglein, HiggsSignals: confronting arbitrary Higgs sectors with measurements at the Tevatron and the LHC, Eur. Phys. J. C 74 (2014) 2711 [arXiv: 1305.1933] [INSPIRE].

[207] G. Bélanger, B. Dumont, U. Ellwanger, J.F. Gunion and S. Kraml, Global fit to Higgs signal strengths and couplings and implications for extended Higgs sectors, Phys. Rev. D 88 (2013) 075008 [arXiv:1306.2941] [inSPIRE].

[208] S. Choi, S. Jung and P. Ko, Implications of LHC data on $125 \mathrm{GeV}$ Higgs-like boson for the Standard Model and its various extensions, JHEP 10 (2013) 225 [arXiv:1307.3948] [INSPIRE].

[209] J. Elias-Miró, J.R. Espinosa, E. Masso and A. Pomarol, Higgs windows to new physics through $d=6$ operators: constraints and one-loop anomalous dimensions, JHEP 11 (2013) 066 [arXiv: 1308.1879] [INSPIRE].

[210] A. Pomarol and F. Riva, Towards the ultimate SM fit to close in on Higgs physics, JHEP 01 (2014) 151 [arXiv: 1308.2803] [INSPIRE].

[211] E. Boos, V. Bunichev, M. Dubinin and Y. Kurihara, Higgs boson signal at complete tree level in the SM extension by dimension-six operators, Phys. Rev. D 89 (2014) 035001 [arXiv: 1309.5410] [INSPIRE].

[212] A. Alloul, B. Fuks and V. Sanz, Phenomenology of the Higgs effective Lagrangian via FeynRules, JHEP 04 (2014) 110 [arXiv:1310.5150] [INSPIRE].

[213] R.L. Delgado, A. Dobado and F.J. Llanes-Estrada, One-loop $W_{L} W_{L}$ and $Z_{L} Z_{L}$ scattering from the electroweak chiral Lagrangian with a light Higgs-like scalar, JHEP 02 (2014) 121 [arXiv: 1311.5993] [INSPIRE].

[214] S. Willenbrock and C. Zhang, Effective field theory beyond the Standard Model, Ann. Rev. Nucl. Part. Sci. 64 (2014) 83 [arXiv:1401.0470] [INSPIRE]. 
[215] C. Englert et al., Precision measurements of Higgs couplings: implications for new physics scales, J. Phys. G 41 (2014) 113001 [arXiv:1403.7191] [INSPIRE].

[216] J. Ellis, V. Sanz and T. You, Complete Higgs sector constraints on dimension-6 operators, JHEP 07 (2014) 036 [arXiv: 1404.3667] [INSPIRE].

[217] E. Masso, An effective guide to beyond the Standard Model physics, JHEP 10 (2014) 128 [arXiv: 1406.6376] [inSPIRE].

[218] A. Biekötter, A. Knochel, M. Krämer, D. Liu and F. Riva, Vices and virtues of Higgs effective field theories at large energy, Phys. Rev. D 91 (2015) 055029 [arXiv:1406.7320] [INSPIRE].

[219] J. de Blas et al., Global Bayesian analysis of the Higgs-boson couplings, Nucl. Part. Phys. Proc. 273-275 (2016) 834 [arXiv: 1410.4204] [INSPIRE].

[220] J. Ellis, V. Sanz and T. You, The effective Standard Model after LHC run I, JHEP 03 (2015) 157 [arXiv: 1410.7703] [INSPIRE].

[221] K. Cheung, J.S. Lee, E. Senaha and P.-Y. Tseng, Confronting Higgcision with electric dipole moments, JHEP 06 (2014) 149 [arXiv:1403.4775] [INSPIRE].

[222] F. Goertz, A. Papaefstathiou, L.L. Yang and J. Zurita, Higgs boson pair production in the $D=6$ extension of the SM, JHEP 04 (2015) 167 [arXiv:1410.3471] [INSPIRE].

[223] J. Bergstrom and S. Riad, Bayesian model comparison of Higgs couplings, Phys. Rev. D 91 (2015) 075008 [arXiv:1411.4876] [INSPIRE].

[224] T. Corbett, O.J.P. Éboli and M.C. Gonzalez-Garcia, Unitarity constraints on dimension-six operators, Phys. Rev. D 91 (2015) 035014 [arXiv:1411.5026] [INSPIRE].

[225] J. Gonzalez-Fraile, Effective Lagrangian approach to the EWSB sector, Nucl. Part. Phys. Proc. 273-275 (2016) 684 [arXiv:1411.5364] [INSPIRE].

[226] K. Cheung, J.S. Lee and P.-Y. Tseng, Higgs data constraints on the minimal supersymmetric Standard Model, Phys. Rev. D 92 (2015) 095004 [arXiv:1501.03552] [INSPIRE].

[227] M. Gorbahn, J.M. No and V. Sanz, Benchmarks for Higgs effective theory: extended Higgs sectors, JHEP 10 (2015) 036 [arXiv:1502.07352] [INSPIRE].

[228] A. Falkowski, Effective field theory approach to LHC Higgs data, Pramana 87 (2016) 39 [arXiv: 1505.00046] [INSPIRE].

[229] A. Falkowski, M. Gonzalez-Alonso, A. Greljo and D. Marzocca, Global constraints on anomalous triple gauge couplings in effective field theory approach, Phys. Rev. Lett. 116 (2016) 011801 [arXiv: 1508.00581] [INSPIRE].

[230] M. Ghezzi, R. Gomez-Ambrosio, G. Passarino and S. Uccirati, NLO Higgs effective field theory and $\kappa$-framework, JHEP 07 (2015) 175 [arXiv: 1505.03706] [INSPIRE].

[231] T. Corbett, O.J.P. Eboli, D. Goncalves, J. Gonzalez-Fraile, T. Plehn and M. Rauch, The Higgs legacy of the LHC run I, JHEP 08 (2015) 156 [arXiv:1505.05516] [INSPIRE].

[232] S. Dwivedi, D.K. Ghosh, B. Mukhopadhyaya and A. Shivaji, Constraints on CP-violating gauge-Higgs operators, Phys. Rev. D 92 (2015) 095015 [arXiv: 1505.05844] [InSPIRE].

[233] S. Fichet and G. Moreau, Anatomy of the Higgs fits: a first guide to statistical treatments of the theoretical uncertainties, Nucl. Phys. B 905 (2016) 391 [arXiv:1509.00472] [InSPIRE]. 
[234] K.D. Gregersen and J.B. Hansen, Frequentist limit setting in effective field theories, arXiv: 1509.01808 [INSPIRE].

[235] C. Englert, R. Kogler, H. Schulz and M. Spannowsky, Higgs coupling measurements at the LHC, Eur. Phys. J. C 76 (2016) 393 [arXiv:1511.05170] [INSPIRE].

[236] A. Drozd, J. Ellis, J. Quevillon and T. You, The universal one-loop effective action, JHEP 03 (2016) 180 [arXiv: 1512.03003] [INSPIRE].

[237] L. Reina et al., Precision constraints on non-standard Higgs-boson couplings with HEPfit, PoS (EPS-HEP2015) 187 [INSPIRE].

[238] A. Butter, O.J.P. Éboli, J. Gonzalez-Fraile, M.C. Gonzalez-Garcia, T. Plehn and M. Rauch, The gauge-Higgs legacy of the LHC run I, JHEP 07 (2016) 152 [arXiv:1604.03105] [INSPIRE].

[239] I. Brivio, J. Gonzalez-Fraile, M.C. Gonzalez-Garcia and L. Merlo, The complete HEFT Lagrangian after the LHC run I, Eur. Phys. J. C 76 (2016) 416 [arXiv:1604.06801] [INSPIRE].

[240] S. Dawson and I.M. Lewis, Singlet model interference effects with high scale UV physics, Phys. Rev. D 95 (2017) 015004 [arXiv:1605.04944] [INSPIRE].

[241] M. Bauer, A. Butter, J. Gonzalez-Fraile, T. Plehn and M. Rauch, Learning from a Higgs-like scalar resonance, Phys. Rev. D 95 (2017) 055011 [arXiv: 1607.04562] [InSPIRE].

[242] A. Freitas, D. López-Val and T. Plehn, When matching matters: loop effects in Higgs effective theory, Phys. Rev. D 94 (2016) 095007 [arXiv: 1607.08251] [INSPIRE].

[243] C. Englert, K. Nordström, K. Sakurai and M. Spannowsky, Perturbative Higgs CP-violation, unitarity and phenomenology, Phys. Rev. D 95 (2017) 015018 [arXiv:1611.05445] [INSPIRE].

[244] M. Ciuchini et al., Updates on fits to electroweak parameters, PoS (LeptonPhoton2015) 013 [INSPIRE].

[245] T. Corbett, A. Joglekar, H.-L. Li and J.-H. Yu, Exploring extended scalar sectors with di-Higgs signals: a Higgs EFT perspective, JHEP 05 (2018) 061 [arXiv:1705.02551] [INSPIRE].

[246] T. Corbett, O.J.P. Éboli and M.C. Gonzalez-Garcia, Unitarity constraints on dimension-six operators II: including fermionic operators, Phys. Rev. D 96 (2017) 035006 [arXiv: 1705. 09294] [INSPIRE].

[247] I. Brivio and M. Trott, The Standard Model as an effective field theory, arXiv:1706.08945 [INSPIRE].

[248] A. Helset and M. Trott, On interference and non-interference in the SMEFT, JHEP 04 (2018) 038 [arXiv: 1711.07954] [InSPIRE].

[249] A. Dedes, M. Paraskevas, J. Rosiek, K. Suxho and L. Trifyllis, The decay $h \rightarrow \gamma \gamma$ in the Standard-Model effective field theory, JHEP 08 (2018) 103 [arXiv: 1805.00302] [INSPIRE].

[250] ATLAS collaboration, Measurement of inclusive and differential cross sections in the $H \rightarrow Z Z^{*} \rightarrow 4 \ell$ decay channel in pp collisions at $\sqrt{s}=13 \mathrm{TeV}$ with the ATLAS detector, JHEP 10 (2017) 132 [arXiv:1708.02810] [INSPIRE].

[251] G. Mancini, Sensitivity studies based on the EFT parametrization in the double differential cross section for the $H \rightarrow Z Z^{*} \rightarrow 4 \ell$ decay channel at LHC, Nuovo Cim. C 39 (2016) 210 [INSPIRE]. 
[252] C. Anastasiou et al., High precision determination of the gluon fusion Higgs boson cross-section at the LHC, JHEP 05 (2016) 058 [arXiv: 1602.00695] [INSPIRE].

[253] T. Sjöstrand et al., An introduction to PYTHIA 8.2, Comput. Phys. Commun. 191 (2015) 159 [arXiv: 1410.3012] [INSPIRE].

[254] S. Ovyn, X. Rouby and V. Lemaitre, DELPHES, a framework for fast simulation of a generic collider experiment, arXiv:0903.2225 [INSPIRE].

[255] J. Alwall et al., The automated computation of tree-level and next-to-leading order differential cross sections and their matching to parton shower simulations, JHEP $\mathbf{0 7}$ (2014) 079 [arXiv: 1405.0301] [INSPIRE].

[256] K. Kondo, Dynamical likelihood method for reconstruction of events with missing momentum. 1: method and toy models, J. Phys. Soc. Jap. 57 (1988) 4126 [InSPIRE].

[257] K. Kondo, Dynamical likelihood method for reconstruction of events with missing momentum. 2: mass spectra for $2 \rightarrow 2$ processes, J. Phys. Soc. Jap. 60 (1991) 836 [INSPIRE].

[258] K. Kondo, T. Chikamatsu and S.H. Kim, Dynamical likelihood method for reconstruction of events with missing momentum. 3: analysis of a CDF high $p_{T} e \mu$ event as $t \bar{t}$ production, $J$. Phys. Soc. Jap. 62 (1993) 1177 [inSPIRE].

[259] R.H. Dalitz and G.R. Goldstein, The decay and polarization properties of the top quark, Phys. Rev. D 45 (1992) 1531 [inSPIRE].

[260] D0 collaboration, B. Abbott et al., Measurement of the top quark mass in the dilepton channel, Phys. Rev. D 60 (1999) 052001 [hep-ex/9808029] [INSPIRE].

[261] J.C. Estrada Vigil, Maximal use of kinematic information for the extraction of the mass of the top quark in single-lepton t $\bar{t}$ events at D0, Ph.D. thesis, FERMILAB-THESIS-2001-07, University of Rochester, Rochester, NY, U.S.A., (2001) [UMI-30-23733] [INSPIRE].

[262] M.F. Canelli, Helicity of the $W$ boson in single-lepton $t \bar{t}$ events, Ph.D. thesis, FERMILAB-THESIS-2003-22, University of Rochester, Rochester, NY, U.S.A., (2003) [UMI-31-14921] [INSPIRE].

[263] D0 collaboration, V.M. Abazov et al., A precision measurement of the mass of the top quark, Nature 429 (2004) 638 [hep-ex/0406031] [INSPIRE].

[264] J.S. Gainer, J. Lykken, K.T. Matchev, S. Mrenna and M. Park, The matrix element method: past, present and future, in Proceedings, 2013 Community Summer Study on the Future of U.S. Particle Physics: Snowmass on the Mississippi (CSS2013), Minneapolis, MN, U.S.A., 29 July-6 August 2013 [arXiv:1307.3546] [INSPIRE].

[265] J.S. Gainer, J. Lykken, K.T. Matchev, S. Mrenna and M. Park, Exploring theory space with Monte Carlo reweighting, JHEP 10 (2014) 078 [arXiv: 1404.7129] [INSPIRE]. 